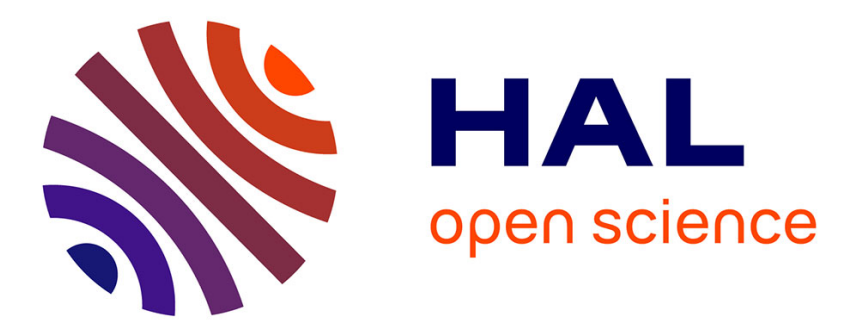

\title{
Theory and analysis of acoustic-gravity waves in a free-surface compressible and stratified ocean
}

Francis Auclair, Laurent Debreu, Emilie Duval, Margot Hilt, Patrick

Marchesiello, Eric Blayo, Franck Dumas, Yves Morel

\section{To cite this version:}

Francis Auclair, Laurent Debreu, Emilie Duval, Margot Hilt, Patrick Marchesiello, et al.. Theory and analysis of acoustic-gravity waves in a free-surface compressible and stratified ocean. Ocean Modelling, 2021, 168, pp.1-20. 10.1016/j.ocemod.2021.101900 . hal-02958158v2

\section{HAL Id: hal-02958158 \\ https://hal.inria.fr/hal-02958158v2}

Submitted on 18 Mar 2021

HAL is a multi-disciplinary open access archive for the deposit and dissemination of scientific research documents, whether they are published or not. The documents may come from teaching and research institutions in France or abroad, or from public or private research centers.
L'archive ouverte pluridisciplinaire HAL, est destinée au dépôt et à la diffusion de documents scientifiques de niveau recherche, publiés ou non, émanant des établissements d'enseignement et de recherche français ou étrangers, des laboratoires publics ou privés. 


\title{
Theory and analysis of acoustic-gravity waves in a free-surface compressible and stratified ocean
}

\author{
F. Auclair*1, L. Debreu ${ }^{2}$, E. Duval ${ }^{2}$, M. Hilt ${ }^{1}$, P. Marchesiello ${ }^{3}$, E. Blayo ${ }^{2}$, F. Dumas ${ }^{5}$, and Y. \\ Morel $^{3}$ \\ ${ }^{1}$ Laboratoire d'Aérologie, Université de Toulouse, CNRS, UPS, France \\ ${ }^{2}$ Univ. Grenoble Alpes, Inria, CNRS, Grenoble INP, LJK, 38000 Grenoble, France \\ ${ }^{3}$ LEGOS, IRD, Université de Toulouse, CNRS, CNES, France \\ ${ }^{5}$ Service Hydrographie et Océanographie de la Marine, Brest, France
}

March 16, 2021

\begin{abstract}
Waves propagate in a free-surface ocean due to compressibility and gravity (and surface tension at much smaller scale). Analytical solutions have long been derived independently for acoustic and gravity waves, i.e., acoustic waves or internal-gravity rays in an unbounded ocean, surface-gravity waves in a free-surface-ocean, and acoustic or internal modes in a bounded ocean. In the present study, surface tension and earth-rotation are neglected and a simple, unified model based on inner and boundary dispersion relations is derived for waves propagating in a compressible, stratified, freesurface ocean. Branches of acoustic gravity wave solutions are identified and visually analysed in phasespace. Taylor developments are then carried out with respect to small parameters describing stratification and compressibility and are compared with numerical approximations of the intersection of inner and boundary dispersion surfaces. Finally, the model recovers the known approximations for swell, longsurface waves, internal-gravity rays, internal modes, acoustic waves or acoustic modes, and also provides modification of these solutions due to stratification and compressibility. Two peculiar regions of the acoustic-gravity wave phase-space are more specifically highlighted and studied in details: one for long waves shedding new light on the distinction between surface waves and low-order internal modes, the other for marginally stable surface waves of intermediate length-scale.
\end{abstract}

*Corresponding author: francis.auclair@aero.obs-mip.fr 


\section{Introduction}

Many types of waves are known to propagate in the ocean, and textbooks (LeBlond and Mysak, 1981; Gill, 1982; Pedlosky, 2013) have detailed the derivation of their analytical solutions for decades. These waves can be classified in several categories depending on the type of mechanisms directly involved in their propagation. Neglecting Earth's rotation and associated planetary waves, two fundamental categories are of particular interest in the present study: acoustic (sound) waves, which are a consequence of ocean compressibility, and gravity waves, which are sustained by the gravity force. Table 1 gives a short (and necessarily incomplete) list of such waves. A particular type of waves is most often characterized by a space-time dispersion relation, linking its time frequency (or period) with its space wave-number (or wavelength). The phase and group velocities and wave dispersion capacity can be derived from the dispersion relations.

\begin{tabular}{l|l|l|l}
\multicolumn{1}{c|}{ Waves } & \multicolumn{1}{c|}{$\begin{array}{c}\text { Assumptions } \\
\text { Angular frequency } \\
(\Omega)\end{array}$} & $\begin{array}{c}\text { Vertical } \\
\text { wave-number } \\
\left(k_{z}\right)\end{array}$ \\
\hline \hline Acoustic waves & $\begin{array}{l}\text { Compressible, } \\
\text { unbounded }\end{array}$ & $\Omega_{a w}^{2}=c_{s}^{2}\left(k_{x}^{2}+k_{z}^{2}\right)$ & independent \\
Internal gravity rays & Stratified, unbounded & $\Omega_{i g r}^{2}=\frac{N^{2} k_{x}^{2}}{k_{x}^{2}+k_{z}^{2}}$ & independent \\
\hline Acoustic gravity modes & Compressible, bounded & $\Omega_{a m}^{2}=c_{s}^{2}\left(k_{x}^{2}+k_{z}^{2}\right)$ & $k_{z, a m}=\frac{\pi}{2 H}+\frac{m \pi}{H}$ \\
Swell & Free-surface & $\Omega_{s w}^{2}=g k_{x} \tanh \left(k_{x} H\right)$ & $k_{z, s w}=k_{x}$ \\
Swell (long) & Free-surface & $\Omega_{l s w}^{2}=g H k_{x}^{2}$ \\
Shallow water waves & Free-surface, shallow & $\Omega_{s h w}^{2}=g H k_{x}^{2}$ & $k_{z, l s w}=k_{x}$ \\
Internal gravity modes & Stratified, bounded & $\Omega_{i m}^{2}=\frac{N^{2} k_{x}^{2}}{k_{x}^{2}+k_{z}^{2}}$ & $k_{z, \text { sh }}=\frac{n \pi}{H}$
\end{tabular}

Table 1: simplified models of ocean waves and modes and their dispersion relations in a vertical section, for an unbounded ocean (top) and for a bounded ocean (bottom). $\Omega$ is the angular frequency of the wave, $k_{x}$ and $k_{z}$ are the horizontal and vertical wave-numbers, $g$ is the acceleration of gravity, $H$ a reference depth, $N$ a reference Brunt-Väisälä angular frequency and $c_{s}$ the speed of sound. $n$ and $m$ are two strictly positive integer numbers.

If ocean waves are short enough and generated far enough from the surface and bottom boundaries, they can propagate in the ocean as in any unbounded medium. However, when their wavelengths are large compared to the ocean depth, ocean waves are known to take specific forms, and the ocean basin is a wave-guide propagating wave modes. For example, the ocean stratification introduces internal gravity modes with "long" horizontal wavelengths. Another example is given by the acoustic modes which are associated with compressibility effects (Jensen et al., 2011) and which have been recently revisited (Smith, 2015). Such internal and acoustic waves are qualified as "modes" because their vertical wavelength is constrained by the vertical extend of the domain. The ocean free-surface is permanently shaken by a myriad of horizontally propagating waves and it is not always clear whether these waves are modes or just vertically-evanescent edge-waves. Capillary waves, swells, tidal waves, tsunamis are well-known examples of such free surface waves.

Deriving a dispersion relation for acoustic waves or for internal wave rays in an unbounded ocean is 
rather straightforward. The method generally includes two steps: small amplitude is assumed; only specific wave-restoring mechanisms and medium characteristics are retained in the simplest possible wave dispersion model (compressibility and pressure force for acoustic waves, gravity and vertical advection of isopycnal surfaces for internal waves). The linear nature of the resulting model has two main advantages: analytical solutions can be derived more easily and waves can be superimposed without interactions (Lighthill, 1967).

The introduction of a free-surface brings more complexity. Small-amplitude is usually postulated in this case also and both gravity and free-surface motions are retained in the dispersion model. However, surface waves are "edge waves" propagating at the interface between the atmosphere and the ocean, and the surface kinematic relation (the free-surface general boundary condition) leads to a transcendental dispersion relation with trigonometric terms. As a result, deriving analytical solutions requires further simplifications. Specific analytical solutions can then be found in the literature depending, for example, on relative depth, i.e., the product of horizontal wave-number $k_{x}$ and ocean depth $H$ (Table 1). Long gravity waves are particular solutions of small aspect ratio $k_{x} H$, well-known to propagate horizontally with $\sqrt{g H}$ phase and group velocities (where $g$ is the acceleration of gravity and $H$ a reference depth). Such waves occur in at least two contexts. Shallow-water waves (SHW) are propagating solutions of shallow-water models, their vertical wave-length is then viewed as infinite (much larger than their horizontal wave-length). Long swells (LSW) are also propagating with the same phase and group velocities but, in this case, they retain some of the properties of swells and their horizontal and vertical wave-lengths have in particular similar magnitudes.

SHW and LSW are often considered as similar since their wave velocity $(\Omega / k x)$ are equal. However due to the underlying assumptions made in the models leading to these solutions, LSW have equal horizontal and vertical wave-numbers $\left(k_{z, l s w}=k_{x}\right)$ whereas SHW have a vanishing vertical wave-number $\left(k_{z, s h w}=0\right)$.

The derivation of mixed acoustic-gravity waves in an ocean bounded by a free surface is even more challenging and is the central subject of this paper. The main objective is to specify the effect of compressibility and stratification on the preceding particular cases of wave solutions. Another target is to found analytical solutions to validate recent developments of the CROCO ocean model, resolving nonhydrostatic equations based on a pseudo-compressible approach (Auclair et al., 2018).

Dukowicz (2013) tackled this problem and proposed a review of "Various approximations in atmosphere and ocean models based on an exact treatment of gravity wave dispersion". In that paper, acousticgravity waves were shown to satisfy a system of two dispersion relations and the impact of several usual assumptions of ocean models was evaluated. The present study builds on Eckart (1960) and Dukowicz (2013)'s results and more specifically focuses on the impact of both stratification and compressibility on acoustic-gravity wave solutions in the ocean: Taylor expansions of dispersion relations and resulting expressions for wavelength and angular frequency are derived in terms of compressibility and stratification. In addition, we show that free-surface can be dealt in an Eulerian framework with the same order of precision as in Dukowicz (2013). A systematic graphic analysis of wave solutions is proposed in 3D angular frequency/wave-number phase-space, unfolding their dependency to the vertical wave-number. The graphic presentation allows to synthesize all possible connections between usual acoustic, internal and surface wave solutions. Surface waves are systematically studied together with internal and acoustic modes. Long-wave solutions are investigated in details and approximate parametric relations are derived for each type of wave. Acoustic modes are discussed in the frame of classical ocean acoustics (Jensen et al., 2011) and additional "poles" (singularities) of the dispersion relations induced by stratification and gravity are found. A peculiar region of phase-space is also identified where surface acoustic-gravity surface wave are "marginally stable" and gravity and compressibility are both important.

In the section 2, a linear model of ocean wave propagation is proposed with bottom and surface boundary conditions, and a corresponding system of two dispersion relations (which will be called inner and boundary dispersion relations) is derived. The inner dispersion relation, which does not take into account the bottom and surface boundary conditions, is studied in details in Section 3, and the wave solutions propagating in an unbounded ocean are investigated. Waves propagating in a bounded ocean, which also 
have to satisfy the boundary relation dispersion, are then studied in Section 4. Conclusions are drawn in Section 5 . 


\section{Linear model for surface and internal acoustic-gravity waves}

\subsection{General model for a compressible, viscous ocean}

Ocean dynamics can be described with a small number of macroscopic variables: velocity (v), pressure and density $(p$ and $\rho$ ), temperature and salinity $(T$ and $S)$. In a Cartesian framework, the general equations governing the motion of a compressible, viscous ocean are then:

$$
\begin{aligned}
\frac{\partial \rho}{\partial t} & =-\nabla \cdot(\rho \mathbf{v}) \\
\frac{\partial \rho \mathbf{v}}{\partial t} & =-\nabla \cdot(\rho \mathbf{v} \otimes \mathbf{v})-2 \rho \mathbf{\Omega} x \mathbf{v}-\nabla p+\nabla \cdot\left(\mu\left(\nabla \mathbf{v}+\nabla \mathbf{v}^{T}\right)+\mu_{2}(\nabla \cdot \mathbf{v}) \mathbf{I}\right)+\rho \mathbf{g} \\
\frac{\partial \rho T}{\partial t} & =-\nabla \cdot(\rho T \mathbf{v})+\nabla \cdot \kappa_{T} \nabla T \\
\frac{\partial \rho S}{\partial t} & =-\nabla \cdot(\rho S \mathbf{v})+\nabla \cdot \kappa_{S} \nabla S \\
\rho \quad & =\rho(T, S, p)
\end{aligned}
$$

where $\mathbf{I}$ is the identity matrix, superscript $\mathrm{T}$ indicates transposition, $\mu$ and $\mu_{2}$ are the kinetic and bulk (or second) viscosities, $\boldsymbol{\Omega}$ is earth angular velocity, $\kappa_{T}$ and $\kappa_{S}$ are the heat and salt diffusivities. These equations are written in a conservative form. They specify basic conservation principles: conservation of mass for Equation (1a), conservation of momentum for Equation (1b) and conservation of heat and salt for equations (1c) and (1d). Equation (1e) is a functional relation describing the thermodynamic equation of state (EOS).

At the bottom $(z=-H)$ and surface $(z=\zeta)$ of the ocean, boundary conditions must be specified for each variable (or for its derivatives). A simple condition of no penetration and no-slip at the ocean flat-bottom can be written:

$$
\mathbf{v}\left(\mathbf{x}_{\mathbf{H}}, z=-H, t\right)=\mathbf{0}
$$

Neglecting surface-tension pressure drop, surface pressure is given by:

$$
p\left(\mathbf{x}_{\mathbf{H}}, z=\zeta, t\right)=p_{\text {atm }}
$$

with $p_{a t m}$ the atmospheric pressure imposed at the surface of the ocean. Surface capillarity waves are consequently filtered out and will be neglected in the remaining of this work.

Relation (2) corresponds to Jensen et al.'s "hard-bottom condition" with no propagation nor penetration of acoustic waves in sediment and geologic layers beneath the ocean floor (Jensen et al., 2011). The authors additionaly recover Relation (3) by assuming that the atmosphere behaves as a vacuum medium for acoustic waves. This means that acoustic waves propagating from the ocean toward the ocean floor or toward the atmosphere are integrally reflected back to the ocean.

The surface kinematic condition expresses the motion of the free-surface and relates the free-surface anomaly $\zeta$ to the surface vertical velocity $w$ :

$$
\frac{\mathrm{d} \zeta\left(\mathbf{x}_{\mathbf{H}}, t\right)}{\mathrm{dt}}=w\left(\mathbf{x}_{\mathbf{H}}, z=\zeta, t\right)
$$

This kinematic boundary condition allows the propagation of surface gravity waves.

\subsection{EOS based on pressure and density decomposition}

Waves are defined as small disturbances to a motionless thermodynamic equilibrium state, and both pressure and density can be decomposed into an equilibrium component and a small increment. In 
addition, as a first approximation, the impact of atmospheric pressure $p_{\text {atm }}$ can be neglected - it can take an active part in wave generation, but only plays a minor role during propagation.

The usual decomposition is now formalized for pressure (5a) and density (5b):

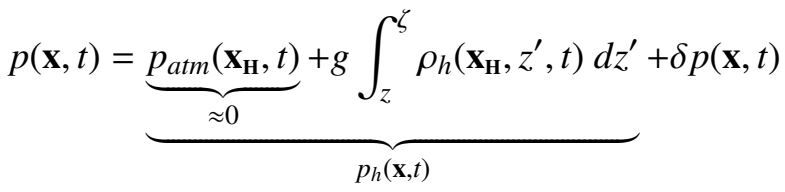

$$
\begin{aligned}
& =\underbrace{g \int_{(\mathbf{x}, t)}^{\zeta} \hat{\rho}_{h}\left(z^{\prime}\right) d z^{\prime}}_{\hat{p}_{h}(z)}+\underbrace{g \int_{z}^{\zeta}\left(\rho_{h}\left(\mathbf{x}_{\mathbf{H}}, z^{\prime}, t\right)-\hat{\rho}_{h}\left(z^{\prime}\right)\right) d z^{\prime}}_{p_{h}^{\prime}(\mathbf{x}, t)}+\delta p(\mathbf{x}, t) \\
& \rho(\mathbf{x}, t)=\underbrace{\hat{\rho}_{T S}(z)+\rho_{T S}^{\prime}(\mathbf{x}, t)}_{\rho_{T S}(\mathbf{x}, t)=\rho(T, S, p=0)}+\underbrace{\frac{1}{c_{s}^{2}}\left(\hat{p}_{h}(z)+p_{h}^{\prime}(\mathbf{x}, t)+\delta p(\mathbf{x}, t)\right)}_{\partial \rho /\left.\partial\right|_{T, S} p(\mathbf{x}, t)}+\mathrm{O}\left(p^{2}\right) \\
& \underbrace{\underbrace{\hat{\rho}_{T S}(z)+\frac{\hat{p}_{h}(z)}{c_{s}^{2}}}_{\hat{\rho}_{h}(z)}+\underbrace{\rho_{T S}^{\prime}(\mathbf{x}, t)+\frac{p_{h}^{\prime}(\mathbf{x}, t)}{c_{s}^{2}}}_{\rho_{h}^{\prime}(\mathbf{x}, t)}}_{\rho_{h}(\mathbf{x}, t)}+\frac{\delta p(\mathbf{x}, t)}{c_{s}^{2}}
\end{aligned}
$$

with $\partial \rho /\left.\partial p\right|_{T, S}=c_{s}^{2}$ at constant temperature and salinity, $\partial \hat{p}_{h} / \partial z=-\hat{\rho}_{h}(z) g, \partial p_{h}^{\prime} / \partial z=-\rho_{h}^{\prime}(z) g$.

The first decomposition $p=p_{h}+\delta p$ is defined by an hydrostatic component $p_{h}$ and a nonhydrostatic pressure increment $\delta p$. It is based on a division of the pressure field into a slow varying component in hydrostatic equilibrium and a fast varying nonhydrostatic component. Density can then be obtained from the EOS (1e) and a first-order Taylor development is carried out for small total pressure. Its Lagrangian evolution can be directly related to the Lagrangian evolution of pressure under the assumption of heat and salt conservation $\left(d \rho_{T S} / d t=0\right)$ :

$$
\frac{\mathrm{d} \rho}{\mathrm{dt}}=\frac{\mathrm{d} \rho_{T S}}{\mathrm{dt}}+\frac{1}{c_{S}^{2}} \frac{\mathrm{d} p}{\mathrm{dt}}=\frac{1}{c_{S}^{2}} \frac{\mathrm{d} p}{\mathrm{dt}}
$$

The Brunt-Väisälä angular frequency for a compressible ocean (Gill (1982), p169) is defined by:

$$
N^{2}=-g / \hat{\rho}_{h}(z) \partial \hat{\rho}_{h} / \partial z-g^{2} / c_{s}^{2}
$$

Note that $\hat{\rho}_{h}(z)$ includes compressibility effects but not $N^{2}$.

\subsection{Linear inviscid, non-rotating wave model}

In the following, Coriolis pseudo-force is neglected whereas viscosities and diffusivities $\mu, \mu_{2}, \kappa_{T}$ and $\kappa_{S}$ are supposed to vanish. Combining mass conservation (1a) and equation of state (8):

$$
-\rho \nabla \cdot \mathbf{v}=\frac{1}{c_{s}^{2}} \frac{\mathrm{d} p}{\mathrm{dt}}
$$

Without loss of generality, the present study can now be restricted to the $(O, x, z)$ vertical plan to simplify notations. Equation (8) can then be expanded to:

$$
-\rho\left(\frac{\partial u}{\partial x}+\frac{\partial w}{\partial z}\right)=\frac{1}{c_{s}^{2}}\left(\frac{\partial p}{\partial t}+u \frac{\partial p}{\partial x}+w \frac{\partial p}{\partial z}\right)
$$


A Taylor expansion of model equations can now be carried out in the vicinity of the reference profiles $\left(\hat{p}_{h}(z), \hat{\rho}_{h}(z)\right)$ and of a resting fluid $(u=w=0)$. Small amplitude wave-induced increments are given by $\delta V=\left(p_{h}^{\prime}+\delta p, \rho_{h}^{\prime}+\delta p / c_{s}^{2}, u, w\right)$. At first order in $\delta V$, conservation of mass and vertical advection of pressure and density can be rewritten. The left-hand side of (9) becomes:

$$
-\rho\left(\frac{\partial u}{\partial x}+\frac{\partial w}{\partial z}\right)=-\left(\frac{\partial \hat{\rho}_{h} u}{\partial x}+\frac{\partial \hat{\rho}_{h} w}{\partial z}-w \frac{\partial \hat{\rho}_{h}}{\partial z}\right)+\mathrm{O}\left(\delta V^{2}\right)
$$

while the right-hand side of (9) becomes:

$$
\frac{1}{c_{s}^{2}}\left(\frac{\partial p}{\partial t}+u \frac{\partial p}{\partial x}+w \frac{\partial p}{\partial z}\right)=\frac{1}{c_{s}^{2}}\left(\frac{\partial p}{\partial t}+w \frac{\partial \hat{p}_{h}}{\partial z}\right)+\mathrm{O}\left(\delta V^{2}\right)=\frac{1}{c_{s}^{2}}\left(\frac{\partial p}{\partial t}-\hat{\rho}_{h} g w\right)+\mathrm{O}\left(\delta V^{2}\right)
$$

leading to:

$$
\frac{\partial p}{\partial t}=-c_{s}^{2}\left(\frac{\partial \hat{\rho}_{h} u}{\partial x}+\frac{\partial \hat{\rho}_{h} w}{\partial z}\right)+\underbrace{\left(g+\frac{c_{s}^{2} \partial \hat{\rho}_{h}(z) / \partial z}{\hat{\rho}_{h}(z)}\right)}_{-c_{s}^{2} N^{2} / g} \hat{\rho}_{h} w+\mathrm{O}\left(\delta V^{2}\right)
$$

At first order in $\delta V$, pressure at $z=0$ can be related to the free-surface anomaly through the hydrostatic relation:

$$
p(z=0)=\hat{\rho}_{h}(0) g \zeta+\mathrm{O}\left(\delta V^{2}\right)
$$

and the kinematic boundary condition can be rewritten for pressure:

$$
\frac{\partial p}{\partial t}(z=0)=g \hat{\rho}_{h}(0) w(z=0)+\mathrm{O}\left(\delta V^{2}\right)
$$

Based on the pressure and density decomposition proposed in $\$ 2.2$, a simpler, inviscid, linear, rotationfree $p-\rho$ model can be used to model acoustic, internal and surface waves. At first order in wave-induced increment $\delta V$, the conservation of momentum and mass and the EOS read:

$$
\begin{aligned}
& \frac{\partial \hat{\rho}_{h} u}{\partial t}=-\frac{\partial p}{\partial x} \\
& \frac{\partial \hat{\rho}_{h} w}{\partial t}=-\frac{\partial p}{\partial z}-\rho g \\
& \frac{\partial \rho}{\partial t}=-\left(\frac{\partial \hat{\rho}_{h} u}{\partial x}+\frac{\partial \hat{\rho}_{h} w}{\partial z}\right) \\
& \frac{\partial p}{\partial t}=-c_{s}^{2}\left(\frac{\partial \hat{\rho}_{h} u}{\partial x}+\frac{\partial \hat{\rho}_{h} w}{\partial z}\right)-\overbrace{\left(\frac{c_{s}^{2}}{D(z)}-g\right)}^{c_{s}^{2} N^{2} / g} \hat{\rho}_{h} w
\end{aligned}
$$

with the (flat) bottom and surface conditions:

$$
\begin{aligned}
& w(z=-H)=0 \\
& \frac{\partial p}{\partial t}(z=0)=\hat{\rho}_{h} g w(z=0)
\end{aligned}
$$

Following Dukowicz (2013), a vertical length scale (written $D(z)$ ) associated with stratification is defined by $D(z)=-\frac{\hat{\rho}_{h}(z)}{\partial \hat{\rho}_{h}(z) / \partial z}=1 /\left(\frac{N^{2}(z)}{g}+\frac{g}{c_{s}^{2}}\right)$. 


\subsection{General propagation equation and polarization}

Form of wave solutions. Dispersion relations can be derived by postulating and specifying the wave form. Horizontally-propagating surface waves, wave modes propagating in the ocean wave guide, internal wave rays and acoustic waves all satisfy the following "polarization" relations:

$$
\left(\begin{array}{c}
\hat{\rho}_{h} u \\
\hat{\rho}_{h} w \\
\rho \\
p
\end{array}\right)=\left(\begin{array}{c}
\widetilde{U}(z) \\
\widetilde{W}(z) \\
\widetilde{\rho}(z) \\
\widetilde{p}(z)
\end{array}\right) e^{i\left(k_{x} x-\Omega t\right)}
$$

where $k_{x}$ and $\Omega$ are respectively the horizontal wave-number and wave angular frequency.

Inner propagation equation \& polarization. Relations (15) can be introduced in the propagation model (13a)-(13d). After some lengthy developments, an ordinary differential equation can be obtained for $\widetilde{W}(z)$ :

$$
\widetilde{W}^{\prime \prime}(z)+\frac{1}{D(z)} \widetilde{W}^{\prime}(z)+\left(k_{x}^{2} \frac{N^{2}-\Omega^{2}}{\Omega^{2}}+\frac{\Omega^{2}}{c_{s}^{2}}-\frac{D^{\prime}(z)}{D^{2}(z)}\right) \widetilde{W}(z)=0
$$

Assuming for now that $\Omega^{2} \neq c_{s}^{2} k_{x}^{2}$ (this particular case is studied in $\S(2.6)$ ), the following polarization relations must be satisfied:

$$
\begin{aligned}
& \widetilde{U}(z)=-i k_{x} \frac{\left(c_{s}^{2}-g D(z)\right) \widetilde{W}(z)+c_{s}^{2} D(z) \widetilde{W}^{\prime}(z)}{D(z)\left(\Omega^{2}-c_{s}^{2} k_{x}^{2}\right)} \\
& \widetilde{\rho}(z)=-i \frac{k_{x}^{2}\left(c_{s}^{2}-g D(z)\right) \widetilde{W}(z)+\Omega^{2} D(z) \widetilde{W}^{\prime}(z)}{D(z) \Omega\left(\Omega^{2}-c_{s}^{2} k_{x}^{2}\right)} \\
& \widetilde{p}(z)=-i \Omega \frac{\left(c_{s}^{2}-g D(z)\right) \widetilde{W}(z)+c_{s}^{2} D(z) \widetilde{W}^{\prime}(z)}{D(z)\left(\Omega^{2}-c_{s}^{2} k_{x}^{2}\right)}
\end{aligned}
$$

Boundary dispersion relations. The polarization relations must also be substituted in the surface boundary condition (14b), leading to:

$$
-i \Omega \widetilde{p}(0)=g \widetilde{W}(0)
$$

or, using (17),

$$
\widetilde{W}^{\prime}(0)+\left(\frac{1}{D(0)}-\frac{g k_{x}^{2}}{\Omega^{2}}\right) \widetilde{W}(0)=0
$$

Note that this differs from the surface boundary condition usualy found in textbook (e.g. (Gill, 1982)) is given by

$$
\widetilde{W}^{\prime}(0)-\left(\frac{g k_{x}^{2}}{\Omega^{2}}\right) \widetilde{W}(0)=0
$$

Equation (19) is identical to Eq. 71 of Dukowicz (2013) who suggests that the term $1 / D(0)$ can only result from governing equations formulated in a Lagrangian vertical coordinate to properly account for the surface boundary condition. Our result contradicts this statement since the term also appears in the Eulerian coordinate system when no incompressibility nor Boussinesq approximation is made.

The boundary condition at the ocean floor is given by:

$$
\widetilde{W}(-H)=0 .
$$


Change of variables. First-order terms can be removed in the ordinary differential equation (16) and further developments can be simplified by making the following change of variable :

$$
\widetilde{W}(z)=\widetilde{W}(0) F(z) e^{\int_{z}^{0} \frac{d z^{\prime}}{2 D\left(z^{\prime}\right)}}
$$

with $F(0)=1$. Substituting this relation in (16) leads to a second-order ordinary differential equation for the unknown function $F(z)$ :

$$
F^{\prime \prime}(z)+\left(k_{x}^{2} \frac{N^{2}-\Omega^{2}}{\Omega^{2}}+\frac{\Omega^{2}}{c_{s}^{2}}-\frac{1+2 D^{\prime}(z)}{4 D(z)^{2}}\right) F(z)=0
$$

$F(z)$ differs from the vertical momentum $\widetilde{W}(z)$ by the attenuation factor $\exp \left(\int_{z}^{0} \frac{\mathrm{d} z^{\prime}}{2 D\left(z^{\prime}\right)}\right)$. This factor reduces the vertical extent of wave anomalies based on the length scale $D(z)$. The weaker the stratification, the larger $D(z)$ and the closer to each other the vertical velocities $\widetilde{W}(z)$ and $F(z)$.

Boundary dispersion relation. In terms of the unknown function $F(z)$, the surface and bottom boundary conditions (19 and 21) reads:

$$
\begin{aligned}
F^{\prime}(0)+\left(\frac{1}{2 D(0)}-\frac{g k_{x}^{2}}{\Omega^{2}}\right) F(0) & =0 \\
F(-H) & =0
\end{aligned}
$$

Constant Brunt-Väisälä angular frequency. In the remaining of the paper, the Brunt-Väisälä angular frequency is assumed to be constant: $N^{2}(z)=N_{0}^{2}$, or equivalently $D(z)=D_{0}$. Equation (22) can then be rewritten:

$$
\widetilde{W}(z)=\widetilde{W}(0) e^{-z / 2 D_{0}} F(z)
$$

and $\hat{\rho}_{h}(z)$ is given by $\hat{\rho}_{h}(z)=\hat{\rho}_{h}(0) e^{-z / D_{0}}$. The general expression of the vertical velocity perturbation profile for a constant scale height $D_{0}$ is thus:

$$
w(x, z, t)=\frac{1}{\hat{\rho}_{h}(z)} \widetilde{W}(z) e^{i\left(k_{x} x-\Omega t\right)}=\frac{\widetilde{W}(0)}{\hat{\rho}_{h}(0)} e^{-z / 2 D_{0}} F(z) e^{i\left(k_{x} x-\Omega t\right)}
$$

Leaving aside for now the surface boundary condition, the vertical profile $F(z)$ has to satisfy the following system of equations:

$$
\begin{array}{r}
F^{\prime \prime}(z)+\overbrace{\left(k_{x}^{2} \frac{N_{0}^{2}-\Omega^{2}}{\Omega^{2}}+\frac{\Omega^{2}}{c_{s}^{2}}-\frac{1}{4 D_{0}^{2}}\right)}^{\equiv k_{z}^{2}} F(z)=0 \\
F(-H)=0
\end{array}
$$

The general solution of (27a)- (27b) with the normalization $F(0)=1$ is

$$
F(z)=\frac{\sin \left(k_{z}(H+z)\right)}{\sin \left(k_{z} H\right)}
$$

where the vertical wave-number $k_{z}$ is defined in (27a) and is a function of $k_{x}$ and $\Omega$. Note that $F(z)$ is defined and linear for $k_{z}=0$, this particular case will be considered separately in $\S(2.6)$. 


\subsection{Inner and boundary dispersion relations}

The relation introduced in (27a) between the vertical wave-number $k_{z}$, horizontal wave-number $k_{x}$ and wave angular frequency $\Omega$ constitutes the first dispersion relation. It is rewritten as:

$$
k_{z}^{2}+k_{x}^{2}\left(1-\frac{N_{0}^{2}}{\Omega^{2}}\right)-\frac{\Omega^{2}}{c_{s}^{2}}+\frac{1}{4 D_{0}^{2}}=0
$$

This relation does not account for surface or bottom boundary conditions and thus only deals with the propagation of waves in the inner ocean. It will now be referred to as the inner dispersion relation. The bottom boundary condition is accounted for in the general solution profile given by (28). Injecting this vertical profile $F(z)$ into the surface boundary condition (27a) then leads to the boundary dispersion relation:

$$
\Omega^{2}=\frac{g k_{x}^{2} \tan \left(H k_{z}\right)}{k_{z}+\frac{\tan \left(H k_{z}\right)}{2 D_{0}}}=\frac{g k_{x}^{2}}{\frac{1}{2 D_{0}}+k_{z} \operatorname{cotan}\left(H k_{z}\right)}
$$

A wave propagating in a "bounded ocean" must satisfy both the inner and boundary (dimensional) dispersion relations (29) and (30). Note that the traditional inner and boundary dispersion relations for a Boussinesq, incompressible fluid (Gill (1982), table 1) can be recovered from (29) and (30) by setting $c_{s} \rightarrow+\infty$ (incompressibility) and then $D_{0} \rightarrow+\infty$ (incompressibility and Boussinesq approximations), leading to:

$$
k_{z}=k_{z, i m}=\frac{n \pi}{H} \quad \text { and } \quad \Omega^{2}=\Omega_{i m}^{2}=N^{2} \frac{k_{x}^{2}}{k_{x}^{2}+k_{z}^{2}}=g k_{x}^{2} \frac{\tan \left(H k_{z}\right)}{k_{z}} \quad \text { for } n \in \mathbb{N}^{*}
$$

Dimensionless dispersion relations. As in Dukowicz (2013), several parameters are now defined to obtain dimensionless dispersion relations:

$$
\epsilon_{i}^{2}=\frac{N^{2} H}{g}, \quad \epsilon_{a}^{2}=\frac{g H}{c_{s}^{2}} \quad \text { and } \quad \epsilon^{2}=\frac{\epsilon_{i}^{2}+\epsilon_{a}^{2}}{2}=-\frac{H}{\hat{\rho}_{h}(z)} \frac{\partial \hat{\rho}_{h}}{\partial z}
$$

$\epsilon_{i}$ is thus a small parameter related to gravity, defined as the ratio of the order of magnitude of the first internal mode $N H$ to the velocity of long surface waves $\sqrt{g H}$. $\epsilon_{a}$ is a small parameter related to acoustics, defined as the ratio of the speed of long surface waves $\sqrt{g H}$ to that of sound waves $c_{s}$. Note that $\epsilon_{i}$ is defined based on the (real, compressible) Brunt-Väisälä angular frequency $N(7)$ to which compressibility effects $\left(g^{2} / c_{s}^{2}\right)$ have been subtracted whereas $\epsilon$ refers to an equivalent stratification where the effects of compressibility are included. In a homogeneous ocean $\epsilon_{i}$ vanishes. The depth scale can then be rewritten as: $D_{0}=2 H / \epsilon^{2}$. This scale-depth does not include any compressibility-induced correction and, as a consequence, it is a function of both small parameters $\epsilon_{i}$ and $\epsilon_{a}$. As a consequence the ratio $H / D_{0}=2 \epsilon^{2}$ give an idea of the relative strength of the ocean stratification.

Three dimensionless variables are also further defined:

$$
\omega=\Omega \sqrt{\frac{H}{g}}, \quad \delta_{x}=k_{x} H, \quad \delta_{z}=k_{z} H
$$

The inner (29) and boundary (30) dispersion relations can be written in terms of the dimensionless parameters and variables as:

$$
\begin{aligned}
\delta_{x}^{2}+\delta_{z}^{2} & =\epsilon_{i}^{2} \frac{\delta_{x}^{2}}{\omega^{2}}+\epsilon_{a}^{2} \omega^{2}-\epsilon^{4} \\
\omega^{2} & =\frac{\delta_{x}^{2} \tan \left(\delta_{z}\right)}{\delta_{z}+\epsilon^{2} \tan \left(\delta_{z}\right)}
\end{aligned}
$$


In a free-surface ocean, wave solutions must satisfy simultaneously relations (34a) and (34b). This means that only one parameter among the angular frequency $\omega$ and horizontal or vertical wave-numbers $\left(\delta_{x}\right.$ and $\left.\delta_{z}\right)$ can be imposed. The other two parameters must adjust for the wave to satisfy the two dispersion relations. For short vertical wave numbers and far from the bottom and surface boundaries, wave solutions only need to satisfy the inner dispersion relation to be dynamically consistent. Pure acoustic waves or pure internal-gravity wave rays are known to propagate in the inner ocean as in an unbounded ocean.

The resulting set of two equations (34a)-(34b) for the three variables $\left(\delta_{x}, \delta_{z}, \omega\right)$ and the two parameters $\epsilon_{a}, \epsilon_{i}$ is nonlinear, and simple general solutions cannot be found analytically.

\subsection{Acoustic Lamb waves \& depth-independent surface gravity waves}

Acoustic Lamb waves. The polarization relations (17) have been derived after excluding the particular dispersion relation:

$$
\epsilon_{a}^{2} \omega^{2}=\delta_{x}^{2}
$$

which is the dispersion relation for acoustic Lamb waves (for atmospheric Lamb waves see for instance Apel (1987)). To be a solution of the linear inviscid, non-rotating wave model (13a)-(13d) with bottom boundary condition (14a), these waves must be of the form:

$$
(\widetilde{W}(z), \widetilde{p}(z), \widetilde{U}(z), \widetilde{\rho}(z))=p_{0}\left(0,1,1 / c_{s}, 1 / c_{s}^{2}\right) e^{-g z / c s^{2}}
$$

Surface pressure must then vanish to satisfy the surface boundary condition (14b) which can only be obtained for the trivial null solution $\left(p_{0}=0\right)$.

This does not mean that Lamb waves cannot propagate in the real ocean but just that they are not solution of the present ocean model (\$2.3). To have non-zero amplitude, the pressure (or alternatively the vertical velocity) must indeed vanish simultaneously at the surface and at the ocean floor. A vanishing pressure both at the surface and at the ocean floor can for instance be found in Jensen's formulation of Pekeris wave guide (Jensen et al., 2011) whereas a vanishing vertical velocity can alternatively be specified by assuming that the present linear inviscid, non-rotating wave model is also rigid lid: (14b) is then replaced by $w(z=0)=0$. In this case, wave polarization is given by (36) with $p_{0}$ the atmospheric pressure at the surface of the ocean.

Depth-independent surface gravity wave. A second interesting wave solution has been identified in $\S(2.4)$ for $\delta_{z}=\delta_{z, 0}=0$. Indeed $F(z)$ is then a linear function of $z$ which simplifies to $F(z)=(z+H) / H$ to satisfy the bottom boundary condition (27b). The surface boundary condition (34b) and the inner dispersion relation (34a) impose that:

$$
\omega^{2}=\omega_{0}^{2}=\frac{\delta_{x, 0}^{2}}{1+\epsilon^{2}}, \quad \delta_{x}^{2}=\delta_{x, 0}^{2}=\frac{\left(1+\epsilon^{2}\right)\left(\epsilon_{i}^{2}+\epsilon^{2}\left(\epsilon_{i}^{2}-\epsilon_{a}^{2}\right) / 2\right)}{1+\left(\epsilon_{i}^{2}-\epsilon_{a}^{2}\right) / 2}=\epsilon_{i}^{2}+O\left(\epsilon_{i}^{4}, \epsilon_{a}^{4}\right)
$$

This wave can only propagate at a well-defined long horizontal wave-length $\delta_{x}=\delta_{x, 0}$ which vanishes in a homogeneous ocean $\left(\epsilon_{i}=0\right)$. It is also depth-independent since if vertical wave-number vanishes.

This depth-independent surface gravity wave presents similarities with Lamb waves (35). Both solutions are found as particular cases while deriving the polarization relations for acoustic-gravity waves and they are associated to an horizontally-propagating convergent-divergent motion of the water column (a depth-independent breathing). This motion is due to the gravity-induced displacement of the ocean for depth-independent surface gravity waves and to compressibility for Lamb waves.

\subsection{Angular frequency is real \& vertical wave-number is either real or pure imaginary}

Based on (23) together with the boundary conditions (24a) and (24b), the vertical wave-number $\delta_{z}$ can be shown to be either real or pure imaginary and the angular frequency $\omega$ can be shown to be real and 
positive under the restrictive assumptions that $\epsilon_{a}=0$ or $\max \left(\epsilon_{i}, \epsilon_{a}\right) \leq \sqrt{2}$. The proof of these properties is detailed in Appendix A.1. It is important to note that $\omega$ is forced to be real by the boundary dispersion relation, and that other solutions are possible when considering the inner dispersion relation only. These solutions will not be discussed in the following, even in Section 3 on waves in an unbounded ocean.

This means that in physically realistic conditions (i.e. $\max \left(\epsilon_{i}, \epsilon_{a}\right) \leq \sqrt{2}$ ) waves are either propagating or evanescent (but not both) along the vertical axis and that they are stable in time: real $-\delta_{z}$ waves are propagating vertically while imaginary- $\delta_{z}$ waves are evanescent vertically. Pure imaginary vertical wave-numbers will be written:

$$
\delta_{z}=i \delta_{z, i} \quad \text { with } \quad \delta_{z, i} \in \mathbb{R}
$$

The inner and boundary dispersion relations (34a) and (34b) write in this case:

$$
\begin{aligned}
\delta_{x}^{2}-\delta_{z, i}^{2} & =\epsilon_{i}^{2} \frac{\delta_{x}^{2}}{\omega^{2}}+\epsilon_{a}^{2} \omega^{2}-\epsilon^{4} \\
\omega^{2} & =\frac{\delta_{x}^{2} \tanh \left(\delta_{z, i}\right)}{\delta_{z, i}+\epsilon^{2} \tanh \left(\delta_{z, i}\right)}
\end{aligned}
$$

When not explicitly mentioned, the standard values of the parameters used in the rest of the paper are listed in Table 2. The rest of the paper is organized as follows: the inner dispersion relation corresponding to waves in an unbounded ocean is studied in Section 3. Additional constraints related to the boundary dispersion relation, i.e., in a bounded ocean, are added in Section 4.

$\begin{array}{lll}\text { Gravity } & g & 9.8 \mathrm{~m} \cdot \mathrm{s}^{-2} \\ \text { Sound speed } & c_{s} & 1500 \mathrm{~m} \cdot \mathrm{s}^{-1} \\ \text { Depth } & H & 4000 \mathrm{~m} \\ \text { Brunt-Väisälä angular frequency } & N=S q r t-\frac{g}{\hat{\rho}_{h}(z)} \frac{\partial \hat{\rho}_{h}}{\partial z}-\frac{g^{2}}{c_{s}^{2}} & 10^{-3} \mathrm{~s}^{-1} \\ \text { Acoustic small parameter } & \epsilon_{a}=\frac{\sqrt{g H}}{c_{s}} & \approx 0.132 \\ \text { Internal small parameter } & \epsilon_{i}=\sqrt{\frac{N^{2} H}{g}} & \approx 0.02020 \\ \text { Equivalent-stratification small parameter } & \epsilon=\sqrt{\frac{\epsilon_{i}^{2}+\epsilon_{a}^{2}}{2}} & \approx 9.4410^{-2} \\ \text { Depth scale } & D_{0}=\frac{H}{2 \epsilon^{2}} & \approx 224 \mathrm{~km}\end{array}$

Table 2: Main parameters used to plot dispersion relations. 


\section{Inner dispersion relation \& waves in an unbounded ocean}

The inner dispersion relation (34a) must be satisfied by any type of ocean waves whether or not the ocean is considered as a locally unbounded medium (far from surface and bottom and for wavelengths small compared to ocean depth). We will show in the present section that (i) in $\left(\delta_{x}, \delta_{z}, \omega\right)$ phase-space, the inner dispersion relation leads to a dispersion surface that can be decomposed in three distinct regions, (ii) two acoustic and stratification reference functions $\omega_{a}\left(\delta_{x}, \delta_{z}\right)$ and $\omega_{i}\left(\delta_{x}, \delta_{z}\right)$ are good approximations of the acoustic and internal regions which in turn correspond to acoustic and internal waves propagating in an unbounded ocean, (iii) the upper and lower regions of the inner dispersion surface (for respectively high and low frequencies) correspond to acoustic waves and internal rays propagating in an unbounded ocean and (iv) the bounded central region of the inner dispersion surface corresponds to vertically vanishing waves, which are referred to as surface waves in $\S(4)$.

\subsection{Acoustic and stratification reference frequencies}

Reference acoustic \& stratification functions. Following Tolstoy (1963), the inner dispersion relation (34a) can be reformulated in the simpler form:

$$
\frac{\omega^{2}}{\omega_{a}^{2}}+\frac{\omega_{i}^{2}}{\omega^{2}}=1
$$

where $\omega_{i}$ and $\omega_{a}$ are functions of horizontal and vertical wave-numbers, defined by:

$$
\begin{aligned}
& \omega_{a}^{2}\left(\delta_{x}, \delta_{z}\right)=\frac{1}{\epsilon_{a}^{2}}\left(\delta_{x}^{2}+\delta_{z}^{2}+\epsilon^{4}\right) \\
& \omega_{i}^{2}\left(\delta_{x}, \delta_{z}\right)=\frac{\delta_{x}^{2} \epsilon_{i}^{2}}{\delta_{x}^{2}+\delta_{z}^{2}+\epsilon^{4}}
\end{aligned}
$$

These two reference functions are not roots of the inner dispersion equation (40) but they are useful in their approximation and, more specifically, in their physical interpretation:

- If $\epsilon_{a}^{2} \omega^{2} \gg \frac{\epsilon_{i}^{2} \delta_{x}^{2}}{\omega^{2}}$ (high angular frequency) then (40) simplifies to $\omega^{2} \approx \omega_{a}^{2}$, relevant to a compressible, homogeneous (unstratified) ocean.

- If $\epsilon_{a}^{2} \omega^{2} \ll \frac{\epsilon_{i}^{2} \delta_{x}^{2}}{\omega^{2}}$ (low angular frequency) then (40) simplifies to $\omega^{2} \approx \omega_{i}^{2}$, relevant to an incompressible, stratified ocean.

Therefore $\omega_{a}$ can be interpreted as a reference acoustic function accounting for the compressibility content of the inner dispersion relation, and is a solution of the inner dispersion relation for an homogeneous ocean. $\omega_{i}$ plays an equivalent role for ocean stratification: it can be interpreted as a reference stratification function and is solution of the inner dispersion relation for an incompressible ocean.

Recall that for $\epsilon_{a}=0$ or $\max \left(\epsilon_{i}, \epsilon_{a}\right) \leq \sqrt{2}$ the angular frequency $\omega$ is real and $\delta z$ is either real or purely imaginary (Appendix A.1). In addition, $\omega_{a}^{2}$ and $\omega_{i}^{2}$ have the same sign, since their product is equal to $\delta_{x}^{2} \frac{\epsilon_{i}^{2}}{\epsilon_{a}^{2}}>0$. Therefore (40) implies that $\omega_{a}^{2} \geq 0, \omega_{i}^{2} \geq 0$, and that:

$$
0 \leq \omega_{i}^{2}\left(\delta_{x}, \delta_{z}\right) \leq \omega^{2}\left(\delta_{x}, \delta_{z}\right) \leq \omega_{a}^{2}\left(\delta_{x}, \delta_{z}\right) \quad \forall\left(\delta_{x}, \delta_{z}\right)
$$

As a consequence, the angular frequency $\omega$ is always bounded by the reference functions $\omega_{i}$ and $\omega_{a}$. 


\subsection{Roots of the inner dispersion relation $\left(\omega_{ \pm}\right)$}

Let us now define $R\left(\delta_{x}, \delta_{z}\right)$ the ratio of stratification to acoustic reference functions:

$$
R^{2}\left(\delta_{x}, \delta_{z}\right)=\frac{\omega_{i}^{2}}{\omega_{a}^{2}}=\frac{\epsilon_{a}^{2} \epsilon_{i}^{2} \delta_{x}^{2}}{\left(\delta_{x}^{2}+\delta_{z}^{2}+\epsilon^{4}\right)^{2}}
$$

$R\left(\delta_{x}, \delta_{z}\right)$ is an important parameter for locating the roots of the inner dispersion relation. As shown in Appendix A.1, $\omega$, the roots of Equation (40), can only be real, a consequence is that $R^{2} \leq \frac{1}{4}$. These roots can then be formulated for the squared angular frequency:

$$
\omega_{ \pm}^{2}=\frac{\omega_{a}^{2}}{2}\left(1 \pm \sqrt{1-4 \frac{\omega_{i}^{2}}{\omega_{a}^{2}}}\right)=\frac{\omega_{a}^{2}}{2}\left(1 \pm \sqrt{1-4 R^{2}}\right)
$$

When $R^{2}$ is small, the two roots are close to the acoustic and stratification reference functions: $\omega_{+} \approx$ $\omega_{a}, \omega_{-} \approx \omega_{i}$. Since their product $\omega_{-}^{2} \omega_{+}^{2}$ is always equal to $\omega_{a}^{2} \omega_{i}^{2}=\frac{\epsilon_{i}^{2}}{\epsilon_{a}^{2}} \delta_{x}^{2}$, we further have:

$$
0 \leq \omega_{i}^{2}\left(\delta_{x}, \delta_{z}\right) \leq \omega_{-}^{2}\left(\delta_{x}, \delta_{z}\right) \leq \frac{\epsilon_{i}}{\epsilon_{a}} \delta_{x} \leq \omega_{+}^{2}\left(\delta_{x}, \delta_{z}\right) \leq \omega_{a}^{2}\left(\delta_{x}, \delta_{z}\right) .
$$

Note also that for a weakly compressible ocean $\left(\epsilon_{a} \ll 1\right)$ :

$$
\omega_{-}^{2}-\omega_{i}^{2}=-\frac{256 \epsilon_{a}^{4} \epsilon_{i}^{6} \delta_{x}^{2}}{\left(\delta_{x}^{2}+\delta_{z}^{2}+\epsilon_{i}^{4}\right)^{5}}+\mathrm{O}\left(\epsilon_{i}^{6}\right)
$$

and for a weakly stratified ocean $\left(\epsilon_{i} \ll 1\right.$ and $\left.\epsilon \ll 1\right)$ :

$$
\omega_{+}^{2}-\omega_{a}^{2}=\frac{\epsilon_{a}^{2} \epsilon_{i}^{4}}{\left(\delta_{x}^{2}+\delta_{z}^{2}+\epsilon_{a}^{4} / 4\right)^{3}}+\mathrm{O}\left(\epsilon_{a}^{6}\right)
$$

\subsection{Three regions in $\left(\delta_{x}, \delta_{z}, \omega\right)$ phase-space}

Figure 1 shows variations of the squared vertical wave-number $\delta_{z}^{2}$ as a function of $\left(\delta_{x}, \omega\right)$ for the values of $\epsilon_{i}, \epsilon_{a}$ given in table (2). Negative values are encountered for medium-range frequencies $\left(10^{-1.7}<\omega<\right.$ $\left.10^{0.7}\right)$ and large enough horizontal wave-numbers $\left(\delta_{x} \geq 0.1-0.2\right)$. This region is surrounded by high and low angular frequency regions of positive $\delta_{z}^{2}$. The transition lines between these regions are given by $\delta_{z}=0$ :

- If $\omega \gg 1$ and $\epsilon_{a} \neq 0$ then $\omega^{2} \approx \omega_{a}^{2}$ and the acoustic transition line is given by $\epsilon_{a}^{2} \omega^{2} \approx \delta_{x}^{2}+\epsilon^{4}$. This line is a parabola and the angular frequency is not bounded when $\delta_{x}$ increases.

- If $\omega \ll 1$ and $\epsilon_{i} \neq 0$ then $\omega^{2} \approx \omega_{i}^{2}$, the equation of the gravity transition line is $\omega^{2} \approx \delta_{x}^{2} \epsilon_{i}^{2} /\left(\delta_{x}^{2}+\epsilon^{4}\right)$. This line has an upper bound $\omega_{c, i}=\epsilon_{i}$. This parabola crosses the $\delta_{x}=0$-axis for the acoustic cut-off angular frequency $\omega_{c, a}=\epsilon^{2} / \epsilon_{a}$. In dimensional form, this bound can be rewritten $\Omega \leq N$ and is related to the well-known cut-off angular frequency for internal gravity waves.

For a real angular frequency $\omega$, Figure (1) confirms that the vertical wave-number can only be real or pure imaginary and that the inner dispersion relation (34a) authorizes three types of wave solutions: two with real vertical wave-numbers $\left(\delta_{z}^{2} \geq 0\right)$ and one with purely imaginary wave-numbers $\left(\delta_{z}^{2}<0\right)$. In this latest region, the corresponding wave solution is evanescent. 


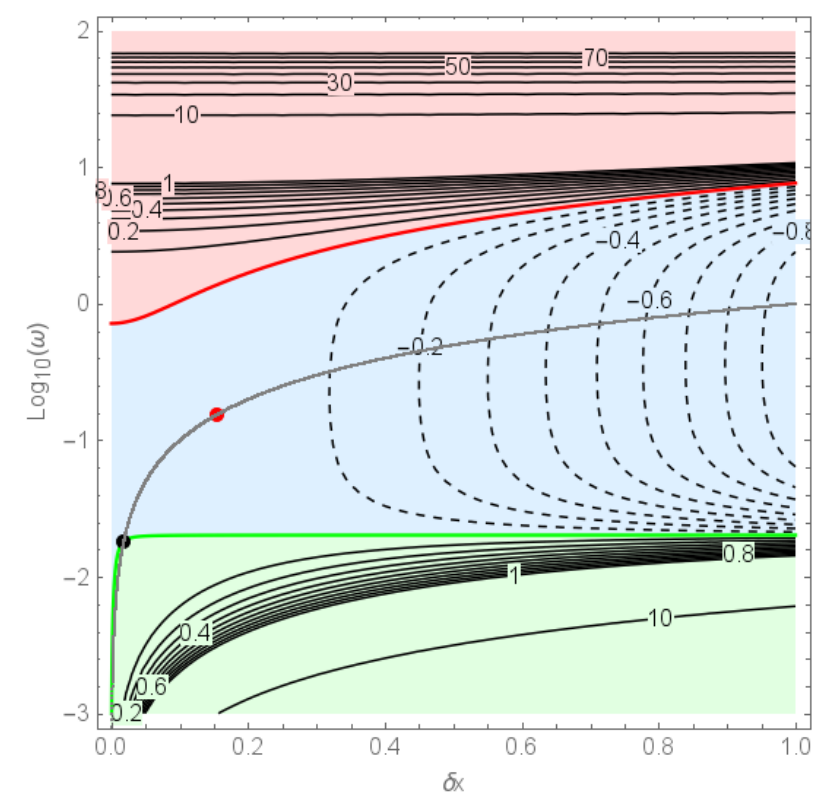

Figure 1: contours of $\delta_{z}^{2}\left(\delta_{x}, \omega\right)$. Plain lines represent positive values $\left(\delta_{z} \in \mathbb{R}\right)$, and dashed lines negative values $\left(\delta_{z} \in i \mathbb{R}\right)$. Background color: (light red) Modified Acoustic Waves-MAW-, (light blue) Modified Surface Waves -MSW-, light (green) Modified Internal Wave rays -MIW-. Black point: barotropic wave solution $\S(2.6)$. Red Point: marginally stable $M S W \$(4.5)$. Red and green curves: acoustic and gravity transition lines $\$(3.3)$. See text below for the definition of the different types of waves.

Separation of solutions. We prove in Appendix (A.2) that, for a non-trivial (non-vanishing) wave solution in a stratified ocean, $R^{2}\left(\delta_{x}, \delta_{z}\right)$ can be equal to $1 / 4$ only if $\delta_{z}$ is pure imaginary. In this case, $R^{2}\left(\delta_{x}, \delta_{z}\right)=1 / 4$ leads to (A.19) and (A.20):

$$
\begin{aligned}
& \delta_{z}^{2}=-\delta_{z, i, *}^{2}=-\delta_{x}^{2}-\epsilon^{4}+2 \epsilon_{a} \epsilon_{i} \delta_{x} \\
& \omega^{2}=\omega_{-}^{2}=\omega_{+}^{2}=\frac{\epsilon_{i}}{\epsilon_{a}} \delta_{x}=\frac{\omega_{a}^{2}}{2}
\end{aligned}
$$

A consequence is that wave solutions are well-separated when $\delta_{z}$ is real (and does not vanish) or when $\delta_{z}$ is pure imaginary and $\delta_{z, i}<\delta_{z, i, *}$. Even if (39a) has two roots in this case, the two corresponding branches are always connected for $\left(\delta_{z}^{2}, \omega^{2}\right)$ satisfying (47a)-(47b), and thus form a single family of ocean waves.

\subsection{Wave solutions in an unbounded ocean}

For a real vertical wave-number $\left(\delta_{z} \in \mathbb{R}\right)^{1}$, the two roots are thus always well-separated and given by:

$$
\omega_{-}\left(\delta_{x}, \delta_{z}\right) \approx \omega_{i}\left(\delta_{x}, \delta_{z}\right), \quad \omega_{+}\left(\delta_{x}, \delta_{z}\right) \approx \omega_{a}\left(\delta_{x}, \delta_{z}\right)
$$

Modified internal waves (MIW). The traditional dispersion relation for dispersive internal gravity wave rays in the context of a Boussinesq incompressible fluid (Gill 1982; see also Table 1 above) is:

$$
\omega^{2}=\omega_{i g r}^{2}=\epsilon_{i}^{2} \frac{\delta_{x}^{2}}{\delta_{x}^{2}+\delta_{z}^{2}}
$$

\footnotetext{
${ }^{1}$ Pure imaginary vertical wave-numbers $\left(\delta_{z} \in i \mathbb{R}\right)$ are treated in $\S(4.4)$ for a bounded ocean.
} 
A Taylor expansion of the gravity-wave root $\omega_{-}^{2}$ given by (43) with respect to the small parameters $\epsilon_{a}$ and $\epsilon_{i}$ leads to:

$$
\begin{aligned}
\frac{\omega_{-}^{2}}{\omega_{i g r}^{2}} & =1-\left(\frac{\epsilon^{4}}{\delta_{x}^{2}+\delta_{z}^{2}}-\frac{\epsilon_{i}^{2} \epsilon_{a}^{2} \delta_{x}^{2}}{\left(\delta_{x}^{2}+\delta_{z}^{2}\right)^{2}}\right)+\mathrm{O}\left(\epsilon^{8}\right) \\
& =1-\underbrace{\frac{\epsilon^{4} \delta_{z}^{2}+4\left(\epsilon_{a}^{2}-\epsilon_{i}^{2}\right)^{2} \delta_{x}^{2}}{\left(\delta_{x}^{2}+\delta_{z}^{2}\right)^{2}}}_{\mathrm{O}\left(\epsilon^{4}\right)}+\mathrm{O}\left(\epsilon^{8}\right),
\end{aligned}
$$

while the development of $\omega_{i}^{2}$ leads to

$$
\frac{\omega_{i}^{2}}{\omega_{i g r}^{2}}=1-\underbrace{\frac{\epsilon^{4}}{\delta_{x}^{2}+\delta_{z}^{2}}}_{\mathrm{O}\left(\epsilon^{4}\right)}+\mathrm{O}\left(\epsilon^{8}\right) .
$$

Compared with $\omega_{i}^{2}, \omega_{-}^{2}$ includes corrective terms confirming that the two roots of the inner dispersion relation are not fully separated. The corrective term $\frac{\epsilon_{i}^{2} \epsilon_{a}^{2} \delta_{x}^{2}}{4\left(\delta_{x}^{2}+\delta_{z}^{2}\right)^{2}}$ is naturally close to $R^{2}$. Formulation (51) shows that the combined effect of compressibility and stratification is always a reduction of the angular frequency, compared with the approximated value $\omega_{i g r}: \omega_{-}^{2} \leq \omega_{i g r}^{2}$.

Ocean waves satisfying (50) will now be referred to as Modified Internal Waves (MIW).

Modified acoustic waves (MAW). The well-known dispersion relation for acoustic waves in an homogeneous fluid is (Table 1):

$$
\omega_{a w}^{2}=\frac{1}{\epsilon_{a}^{2}}\left(\delta_{x}^{2}+\delta_{z}^{2}\right)
$$

A Taylor development of the acoustic root $\left(\omega_{+}\right)$with respect to $\epsilon_{a}$ and $\epsilon_{i}$ leads this time to:

$$
\begin{aligned}
\frac{\omega_{+}^{2}}{\omega_{a w}^{2}} & =1+\left(\frac{\epsilon^{4}}{2\left(\delta_{x}^{2}+\delta_{z}^{2}\right)}-\frac{\epsilon_{i}^{2} \epsilon_{a}^{2} \delta_{x}^{2}}{\left(\delta_{x}^{2}+\delta_{z}^{2}\right)^{2}}\right)+\mathrm{O}\left(\epsilon^{8}\right) \\
& =1+\underbrace{\frac{\epsilon^{4} \delta_{z}^{2}+\left(\epsilon_{a}^{2}-\epsilon_{i}^{2}\right)^{2} \delta_{x}^{2}}{2\left(\delta_{x}^{2}+\delta_{z}^{2}\right)^{2}}}_{\mathrm{O}\left(\epsilon^{4}\right)}+\mathrm{O}\left(\epsilon^{8}\right),
\end{aligned}
$$

while the development of $\omega_{a}^{2}$ leads to

$$
\frac{\omega_{a}^{2}}{\omega_{a w}^{2}}=1+\underbrace{\frac{\epsilon^{4}}{\delta_{x}^{2}+\delta_{z}^{2}}}_{\mathrm{O}\left(\epsilon^{4}\right)}+\mathrm{O}\left(\epsilon^{8}\right) .
$$

Compared with $\omega_{a}^{2}, \omega_{+}^{2}$ includes corrective terms due to the two roots of the inner dispersion relation not being fully separated. Again, the corrective term is small and close to $R^{2}$. The combined effect of compressibility and stratification is always an increase of the angular frequency, compared with the approximated value $\omega_{a w}: \omega_{+}^{2} \geq \omega_{a w}^{2}$.

Ocean waves satisfying (53) will be called Modified Acoustic Waves (MAW) in the following. The modifications to usual internal and acoustic wave dispersion relations by compressibility and stratification effects are expressed by:

$$
\omega_{+}^{2} \omega_{-}^{2}=\frac{\epsilon_{i}^{2}}{\epsilon_{a}^{2}} \delta_{x}^{2}=\omega_{a w}^{2} \omega_{i g r}^{2},
$$

which can explain the symmetry in the above developments for modified internal and acoustic waves. 


\subsection{Homogeneous and/or incompressible unbounded ocean}

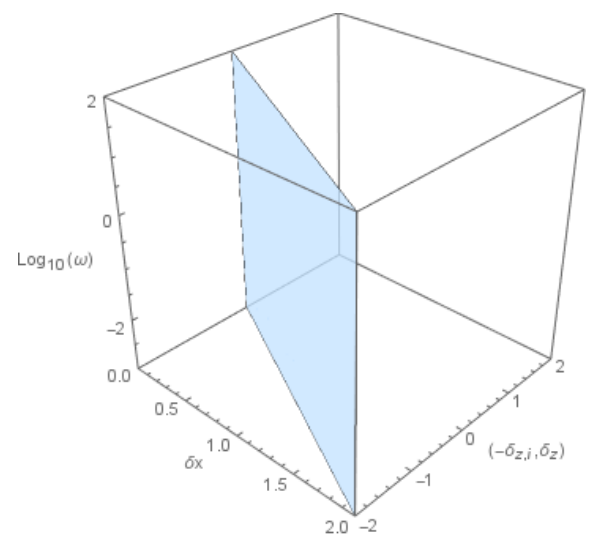

(a)

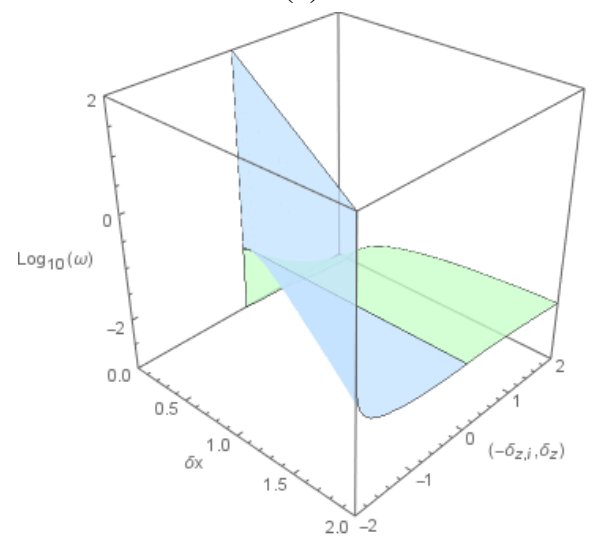

(c)

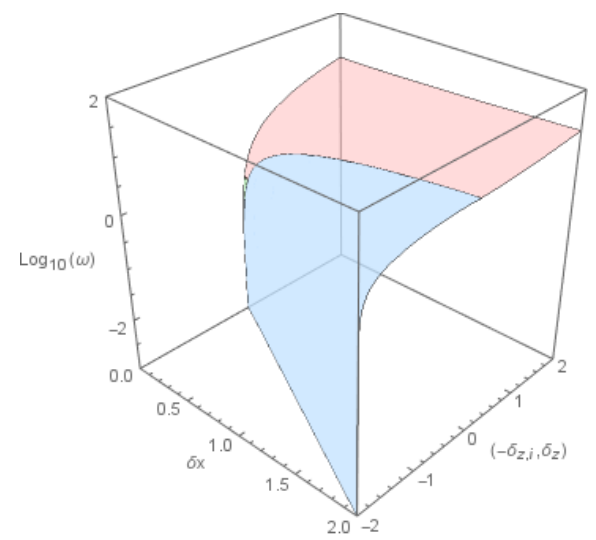

(b)

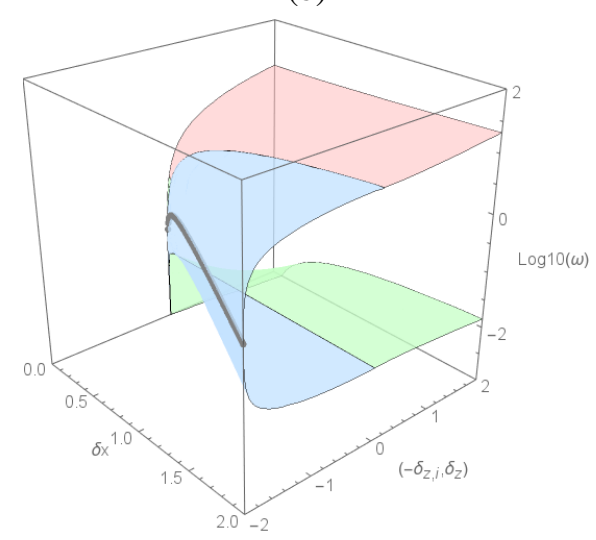

(d)

Figure 2: inner dispersion surfaces in $\left(\delta_{x}, \delta_{z}, \omega\right)$ space.

(a) homogeneous, incompressible ocean $\left(\epsilon_{i}=\epsilon_{a}=0\right)$,

(b) homogeneous, compressible ocean $\left(\epsilon_{i}=0\right)$,

(c) stratified, incompressible ocean $\left(\epsilon_{i}=0\right)$,

(d) stratified, compressible ocean $\left(\epsilon_{a}=0\right)$.

Colors: (light red) acoustic wave region $\left(\delta_{z}^{2} \geq 0 \mathcal{E} \omega\right.$ large), (light blue) surface wave region $\left(\delta_{z}^{2} \geq 0 \mathcal{E}\right.$ $\omega$ small), (light green) internal wave region $\left(\delta_{z}^{2}<0\right)$. Gray curve: triplets $\left(\delta_{x}, \delta_{z}, \omega\right)$ satisfying $(47 b)$ and (47a). Negative values of the $\delta_{z}$ axis correspond to $-\delta_{z, i}$, i.e. minus the imaginary part of pure imaginary vertical wave-numbers.

Much insight can be gained on acoustic-gravity waves by a geometrical investigation of surfaces (in phase space) that correspond to the inner and boundary dispersion relations. These surfaces will respectively be named inner and boundary dispersion surfaces.

Figure (2) shows the inner dispersion surfaces in the $\left(\delta_{x}, \delta_{z}, \omega\right)$ space. Each dispersion surface corresponds to a region of the wave solutions. These surfaces are plotted for a homogeneous and incompressible (2.a), a homogeneous and compressible (2.b), an incompressible and stratified (2.c) and a compressible and stratified unbounded ocean. The y-axis is constructed such that the $\left(\delta_{z}>0\right)$ positive half-space corresponds to the real vertical wave-numbers whereas the $\left(\delta_{z}<0\right)$ negative half-space corresponds to the (imaginary part of) pure-imaginary vertical wave-numbers $\left(\delta_{z, i}\right)$.

Careful inspection of the $\left(\delta_{z}>0\right)$ half space in figures (2.b), (2.c) and (2.d) confirms the presence of two regions of solutions with real positive vertical wave-numbers. For large angular frequencies (red branch), the upper region corresponds to acoustic wave solutions (MAW) (figures 2.b and 2.d). This region disappears under the assumption of incompressibility (figues 2.a and 2.c). The lower region (light-green branch) corresponds to internal wave solutions (MIW) (figures 2.c and 2.d) and disappears 
under the assumption of homogeneous ocean (figures 2.a and 2.b).

The third region of wave-solution (light-blue surfaces in figure 2) corresponds to pure-imaginary vertical wave-numbers. In a stratified, compressible, unbounded ocean (figure 2.d), this region corresponds to intermediate values of the .

In a homogeneous, incompressible and unbounded ocean, figure (2.a) shows that the unique dispersion surface is the $\left(\delta_{x}^{2}=-\delta_{z}^{2}\right)$ plane.

\subsection{Summary: waves solutions in an unbounded ocean}

In the preceding analysis, three types of waves were identified. A synthesis is given by figure $2 . \mathrm{d}$ showing the inner dispersion surfaces for a stratified, compressible ocean and figure 2.a-c showing the limit cases for respectively a homogeneous and incompressible, a homogeneous and compressible and a stratified but incompressible ocean. Approximate frequency values for modified internal and acoustic waves are summarized in Table (3) and Table (4) in dimensional form, for comparison with introductory Table (1). Since the practical existence and characterization of modified surface waves is totally dependent on boundary conditions, they are not summarized here but will be detailed in the next section.

\begin{tabular}{l|l|l} 
& Internal Waves & Acoustic Waves \\
\hline a) $\left(\epsilon_{i}=\epsilon_{a}=0\right)$ & - & - \\
b) $\left(\epsilon_{i}=0, \epsilon_{a} \neq 0\right)$ & - & $\frac{\omega_{+}^{2}}{\omega_{a w}^{2}} \approx 1+\frac{\epsilon_{a}^{4}}{4\left(\delta_{x}^{2}+\delta_{z}^{2}\right)}$ \\
c) $\left(\epsilon_{i} \neq 0, \epsilon_{a}=0\right)$ & $\frac{\omega_{-}^{2}}{\omega_{i w r}^{2}} \approx 1-\frac{\epsilon_{i}^{4}}{4\left(\delta_{x}^{2}+\delta_{z}^{2}\right)}$ & - \\
d) $\left(\epsilon_{i} \neq 0, \epsilon_{a} \neq 0\right)$ & $\frac{\omega_{-}^{2}}{\omega_{i w r}^{2}} \approx 1-\frac{\left(\epsilon_{i}^{2}+\epsilon_{a}^{2}\right)^{2} \delta_{z}^{2}+\left(\epsilon_{a}^{2}-\epsilon_{i}^{2}\right)^{2} \delta_{x}^{2}}{4\left(\delta_{x}^{2}+\delta_{z}^{2}\right)^{2}}$ & $\frac{\omega_{+}^{2}}{\omega_{a w}^{2}} \approx 1+\frac{\left(\epsilon_{i}^{2}+\epsilon_{a}^{2}\right)^{2} \delta_{z}^{2}+\left(\epsilon_{a}^{2}-\epsilon_{i}^{2}\right)^{2} \delta_{x}^{2}}{4\left(\delta_{x}^{2}+\delta_{z}^{2}\right)^{2}}$
\end{tabular}

Table 3: Modified Internal and Acoustic waves in an unbounded ocean.

\begin{tabular}{l|l} 
Waves & Frequency $(\Omega)$ \\
\hline Modified Acoustic Waves (MAW) & $\Omega_{\text {maw }}^{2}=c_{s}^{2}\left(k_{x}^{2}+k_{z}^{2}\right)\left[1+\frac{1}{4\left(k_{x}^{2}+k_{z}^{2}\right)^{2}}\left(\frac{k_{z}^{2}}{D_{0}^{2}}+\left(\frac{g}{c_{s}^{2}}-\frac{N^{2}}{g}\right)^{2} k_{x}^{2}\right)\right]$ \\
Modified Internal Waves (MIW) & $\Omega_{\text {miw }}^{2}=\frac{N^{2} k_{x}^{2}}{k_{x}^{2}+k_{z}^{2}}\left[1-\frac{1}{4\left(k_{x}^{2}+k_{z}^{2}\right)^{2}}\left(\frac{k_{z}^{2}}{D_{0}^{2}}+\left(\frac{g}{c_{s}^{2}}-\frac{N^{2}}{g}\right)^{2} k_{x}^{2}\right)\right]$
\end{tabular}

Table 4: Compressibility and stratification induced modifications to the usual dispersion relations given in Table (1). $\Omega$ is wave angular frequency, $k_{x}$ and $k_{z}$ are the wavenumbers, $g$ is the acceleration of gravity, $N$ a reference Brunt-Väisälä frequency and $c_{s}$ the speed of sound. $D_{0}$ is the background density vertical scale, given by $1 / D_{0}=N^{2} / g+g / c_{s}^{2}$

In a more realistic bounded ocean, their existence is guaranteed only if their vertical scale is (much) smaller than the ocean depth $\left(\left|\delta_{z}\right| \gg 1\right)$ and if they do not interfere with the bottom or the surface of the ocean. The next section will investigate the impact of adding the boundary dispersion relation (34b). 


\section{Waves in a bounded ocean}

Acoustic-gravity waves propagating in a bounded ocean (i.e. considering both the free-surface and the bottom boundary of the ocean) are studied in the present section. These wave solutions are first shown to be organised in branches (i.e. in 1D curves parameterized by $\left(\delta_{x}^{2}\left(\delta_{z}\right), \omega^{2}\left(\delta_{z}\right)\right)$. Each branch is located at the intersection of the inner and boundary dispersion surfaces and is confined to a region of phase space located between two poles of the dispersion relations.

Modified internal and acoustic modes and then modified surface waves are studied before acousticgravity waves in the long wave approximation are investigated.

\subsection{Graphical investigation of Modified Surface Waves (MSW), Modified Acoustic Modes (MAM) and Modified Internal Modes (MIM)}

The compressible and stratified ocean is now assumed to be bounded. Wave solutions must thus satisfy both the inner (34a) and boundary (34b) dispersion relations. In phase space, they must lie at the intersections of the inner and boundary dispersion surfaces, which are now plotted simultaneously on figure 3. For real vertical wave-numbers $\left(k_{z} \in \mathbb{R}\right.$, Figure $\left.3 \mathrm{a}\right)$, the boundary dispersion surface is a

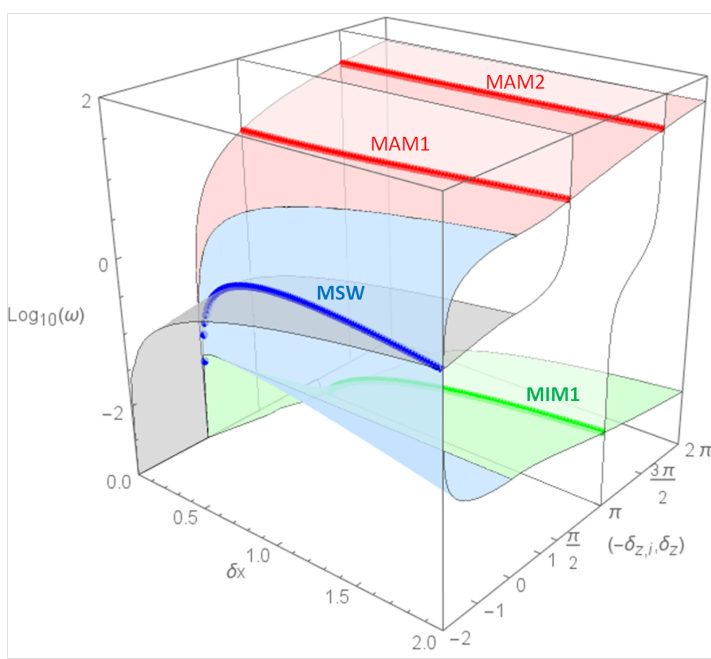

(a)

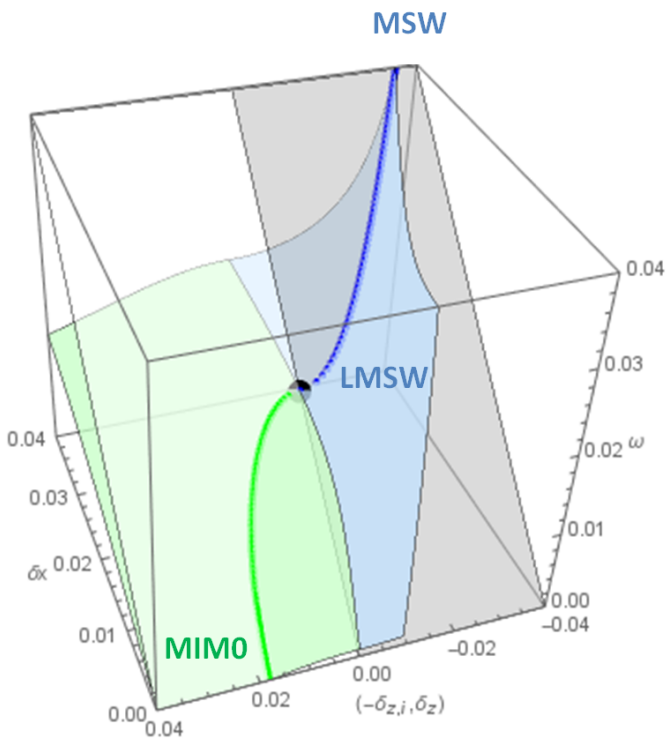

(b)

Figure 3: inner and boundary dispersion surfaces in $\left(\delta_{x}, \delta_{z}, \omega\right)$ space and wave solutions for short and intermediate wavelengths (a) and for long waves (b). Colors: (light red) acoustic wave region $\left(\delta_{z}^{2} \geq 0 \mathcal{E}\right.$ $\omega$ large), (light blue) surface wave region ( $\delta_{z}^{2} \geq 0 \mathcal{E} \omega$ small), (light green) internal wave region $\left(\delta_{z}^{2}<0\right)$, (white) boundary dispersion surface for $\delta_{z} \in \mathbb{R}$, (light gray) boundary dispersion surface for $\delta_{z} \in \mathbb{R}$. Blue curve: Modified Surface Waves (MSW). Red curves: Modified Acoustic Modes (MAM). Green curve: Modified Internal Modes (MIM). Black point: depth-independent surface gravity wave\$(2.6).

piecewise surface. Several regions that are nearly vertical in $\left(\delta_{x}, \delta_{z}, \log _{10}(\omega)\right)$ space at $\delta_{z} \approx n \pi$ (small $\omega)$ and at $\delta_{z} \approx \pi / 2+(m-1) \pi$ (large $\omega$ ) with $n \in \mathbb{N}^{*}$ and $m \in \mathbb{N}^{*}$. The intersection of these surfaces with inner dispersion relation surfaces result in a number of constrained vertical wave-numbers (according to $n$ and $m$ ). We will show in the following sections that the resulting wave solutions are "modes" and the two intersections correspond more specifically to Modified Internal Modes (MIM), $n \in \mathbb{N}^{*}$ and to Modified Acoustic Modes (MAM), $m \in \mathbb{N}^{*}$.

For purely imaginary wave-numbers, the (light blue) inner dispersion surface looks like an horizontal hyperbolic surface which intersects the (light gray) boundary dispersion surface. The resulting wave 
solutions correspond to Modified Surface Waves $(M S W)$. Far from the origin $\left(\delta_{x}, \delta_{z}\right)=(0,0)$, at the intersection points, $\left|\delta_{z}\right|$ is close to $\delta_{x}$.

For long waves $\left(\delta_{x} \ll 1 \&\left|\delta_{z}\right| \ll 1\right)$, the boundary dispersion surfaces for real and purely imaginary $\delta_{z}$ are in the same plane. Indeed, the development of the boundary relation is well approximated by $\omega^{2} \approx \delta_{x}^{2}$ in both cases (a better approximation is given in Eq. 65). Figure 3b shows the different solutions close to the origin $\left(\delta_{x}, \delta_{z}, \omega\right)=(0,0,0)$. The acoustic-wave surface does not intersect the boundary dispersion surface near the origin (as proven in $\S 4.5$ ) and is not present close to the origin.

In the context of a bounded ocean, three types of wave solutions are thus graphically identified, spreading on the three regions of the inner dispersion surface, while satisfying the boundary dispersion relation: internal gravity (in a stratified ocean), acoustic (in a compressible ocean) and surface waves (in a freesurface ocean). They are investigated in the following using Taylor expansions of the general roots $\omega_{ \pm}$, with respect to small parameters $\left(\epsilon_{i}, \epsilon_{a}\right)$, leading to simple approximations of wave dispersion relations. When needed, asymptotic relations are derived with respect to $\delta_{x}, \delta_{z}$ or $\omega$. Taylor expansions will give indications of how usual wave solutions can be modified by gravity and stratification $\left(\epsilon_{i}\right)$, and/or by compressibility $\left(\epsilon_{a}\right)$.

\subsection{Poles of the dispersion relations}

The dispersion relations for acoustic-gravity waves in a bounded ocean exhibit several discrete poles or singularities (for the vertical wave-number) that lead to the existence of normal modes. To show this, we can combine the dispersion relations (34a) and (34b) to express the square of the horizontal wave-number and the angular frequency as functions of the vertical wave number:

$$
\begin{aligned}
& \delta_{x}^{2}\left(\delta_{z}\right)=\frac{\left(\epsilon^{2} \sin \left(\delta_{z}\right)+\delta_{z} \cos \left(\delta_{z}\right)\right)\left(\left(\delta_{z}^{2}+\left(\epsilon_{i}^{4}-\epsilon_{i}^{4}\right) / 4\right) \sin \left(\delta_{z}\right)-\delta_{z} \epsilon_{i}^{2} \cos \left(\delta_{z}\right)\right)}{\left(\left(\epsilon_{a}^{2}-\epsilon_{i}^{2}\right) \sin \left(\delta_{z}\right) / 2-\delta_{z} \cos \left(\delta_{z}\right)\right) \sin \left(\delta_{z}\right)} \\
& \omega_{x}^{2}\left(\delta_{z}\right)=\frac{\left(\delta_{z}^{2}+\left(\epsilon_{a}^{4}-\epsilon_{i}^{4}\right) / 4\right) \sin \left(\delta_{z}\right)-\delta_{z} \epsilon_{i}^{2} \cos \left(\delta_{z}\right)}{\left(\epsilon_{a}^{2}-\epsilon_{i}^{2}\right) \sin \left(\delta_{z}\right) / 2-\delta_{z} \cos \left(\delta_{z}\right)}
\end{aligned}
$$

The first relation (55a) has two poles (or singularities): $\delta_{z}=\delta_{z, n}=n \pi$ with $n \in \mathbb{N}$ is indeed an approximate root of $\sin \left(\delta_{z}\right)=0$ and thus a pole of $\delta_{x}^{2}\left(\delta_{z}\right)$ and $\delta_{z}=\delta_{z, m} \approx r_{m}=\pi / 2+m \pi$ is a root of $\delta_{z} / \tan \left(\delta_{z}\right)=\epsilon_{a}^{2}-\epsilon_{i}^{2} \ll 1$ and thus a pole of $\delta_{x}^{2}\left(\delta_{z}\right)$. The second dispersion relation (55b) only has one pole: $\delta_{z}=\delta_{z, m} \approx r_{m}=\pi / 2+m \pi$. A more accurate expression of $\delta_{z, m}$ can be obtained as an infinite series:

$$
\delta_{z, m}=r_{m}-\frac{\epsilon_{a}^{2}-\epsilon_{i}^{2}}{r_{m}}-\frac{\left(\epsilon_{a}^{2}-\epsilon_{i}^{2}\right)^{2}\left(3+\epsilon_{i}^{2}-\epsilon_{a}^{2}\right)}{3 r_{m}^{3}}-\frac{\left(\epsilon_{a}^{2}-\epsilon_{i}^{2}\right)^{3}\left(3\left(\epsilon_{a}^{2}-\epsilon_{i}^{2}\right)^{2}-20\left(\epsilon_{a}^{2}-\epsilon_{i}^{2}\right)+30\right)}{15 r_{m}^{5}}+\mathrm{O}\left(\frac{1}{r_{m}^{7}}\right)
$$

Note that $\omega^{2}\left(\delta_{z}\right)$ is continuous in $\delta_{z}=\delta_{z, n}$. This set of poles confirms and extends the poles found by Jensen et al. (2011) for a compressible, homogeneous, rigid lid, "ideal ocean waveguide". Indeed, the authors further show that the normal mode expansion of the general integral solution can be written as an infinite sum of the complex residues associated to these poles. $\left(\delta_{z, m}, \delta_{z, n}\right)$ is the extended set of poles of acoustic gravity wave dispersion in a bounded ocean and two types of modes now need to be investigated. We further show in (A.3) that the first type $\left(\delta_{z, m}\right)$ corresponds to the Modified Acoustic Modes (MAM) identified graphically in $\S(4.1)$ and plotted with a red curve in figure (3). The second type $\left(\delta_{z, n}\right)$ is also shown to correspond to the Modified Internal Modes (MIM) also identified graphically in $\$(4.1)$ but plotted in green.

Figure (4) shows that wave solutions are organized in branches confined to the intervals $\left.\delta_{z}^{2} \in\right]-\infty, \delta_{z, m=1}^{2}$ [, $\left.\delta_{z} \in\right] \delta_{z, m=n}, \delta_{z, n}\left[\right.$ and $\left.\delta_{z} \in\right] \delta_{z, n}, \delta_{z, m=n+1}\left[\right.$ for $n \in \mathbb{N}^{*}$.The $\delta_{x}^{2}+\delta_{z}^{2}=0$ vertical plane, coloured in light gray, is the region where the induced effects of compressibility and stratification exactly compensate. Indeed, (34a) implies in this case that $\epsilon_{a}^{2} \omega^{2}+\epsilon_{i}^{2} \delta_{x}^{2} / \omega^{2}-\epsilon=0$. 

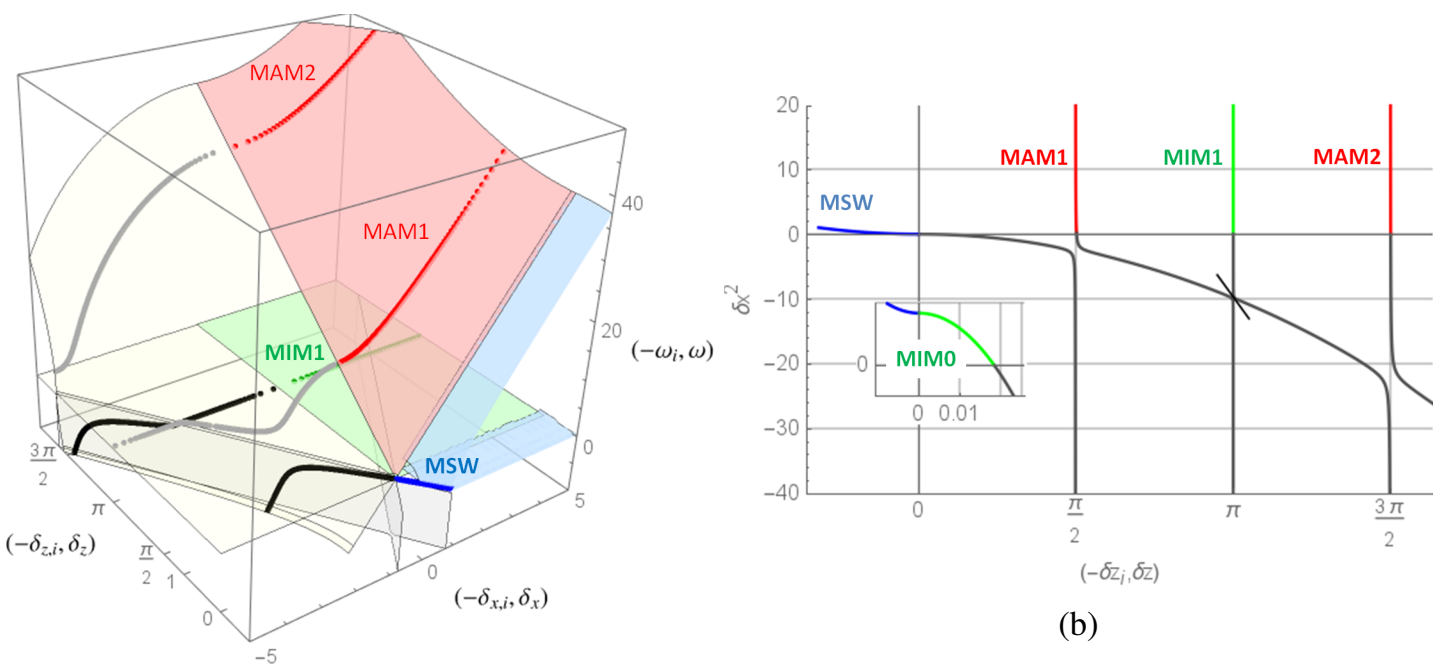

(b)

(a)
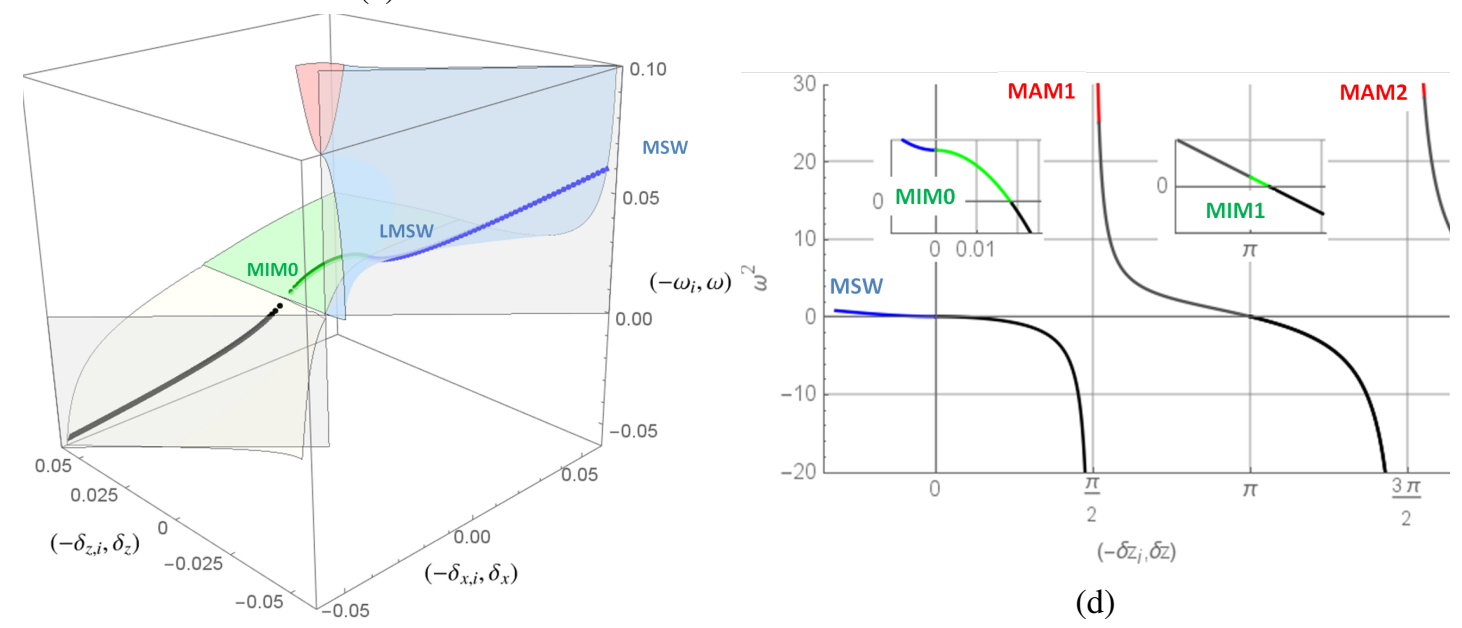

(d)

(c)

Figure 4: (a, c) show the inner and boundary dispersion surfaces in $\left(\delta_{x}, \delta_{z}, \omega\right)$ space and wave solutions for long waves, (b, d) show $\delta_{x}^{2}\left(\delta_{z}\right)$ and $\omega^{2}\left(\delta_{z}\right)$. Coloured surfaces: (light red) acoustic wave region, (light blue) surface wave region, (light green) internal wave region, (light yellow) inner dispersion surface in half space $\delta_{x}^{2}<0$, (light gray) $\delta_{x}^{2}+\delta_{z}^{2}=0$ vertical plane. Acoustic gravity wave branches: MIM0 (green curve), LMSW (blue curve) branch, MIM (green curves), MAM (red curves prolonged in gray when $\delta_{x}^{2}<0$ ), MIM (green curves prolonged in black when $\delta_{x}^{2}<0$ ). Note that wave solutions with pure imaginary horizontal wave-numbers $\left(\delta_{x, i} \in i \mathbb{R}\right)$ or pure imaginary angular frequencies $(\omega \in i \mathbb{R})$ are plotted to clearly identify wave branches. These solutions correspond to waves decaying restively in the horizontal direction and in time and are not further discussed in the present study.

A study of the variations of $\delta_{x}^{2}\left(\delta_{z}\right)$ and $\omega^{2}\left(\delta_{z}\right)$ shows that these functions are monotonically decreasing at respectively the third and first order in $\left(\epsilon_{a}, \epsilon_{i}\right)$ (see appendix §A.7). When $\delta_{z} \rightarrow \delta_{z, m}^{ \pm}, \delta_{x}^{2}\left(\delta_{z}\right) \rightarrow \pm \infty$ and $\omega^{2}\left(\delta_{z}\right) \rightarrow \pm \infty$. When $\delta_{z} \rightarrow \delta_{z, n}^{ \pm}, \delta_{x}^{2}\left(\delta_{z}\right) \rightarrow \pm \infty$ but $\omega^{2}\left(\delta_{z}\right)= \pm \epsilon_{i}^{2}$ since $\delta_{z, n}$ is not a pole of $\omega\left(\delta_{z}\right)$. In the interval ] $-\infty, \delta_{z, m=0}$ ], the long-wave branch extends to negative values of $\delta_{z}^{2}$ (for a pure imaginary vertical wave-number, blue curve in figure 4 . Each branch of the acoustic gravity wave solutions consequently extends both in the $\delta_{x}^{2} \geq 0$ half space of propagating waves (green, red and blue curves in figure 4) and in the $\delta_{x}^{2}<0$ half space of vanishing waves (gray and black curves).

This study of the variations of $\delta_{x}^{2}\left(\delta_{z}\right)$ further shows that for waves propagating both horizontally and vertically $\left(\delta_{x}^{2}, \delta_{z}^{2} \geq 0\right), \delta_{z}$ remains close to either $\delta_{z, m}$ or $\delta_{z, n}$ in each interval of solutions except for the very-long-wave branch (figure 4.c and $0<\delta_{z}^{2}<\delta_{z, m=0} \approx \pi / 2$ ). Indeed, in the case of "not-too-long" propagating waves, $\delta_{x}^{2}+\delta_{z}^{2}$ is positive and at least larger than $\delta_{z, m=0}^{2}$ meaning that, whatever $\delta_{x}$, either 
$\epsilon_{a}^{2} \omega^{2}=\epsilon_{a}^{2} \delta_{x}^{2} /\left(\epsilon^{2}+\delta_{z} \cot \left(\delta_{z}\right)\right)$ or $\epsilon_{i}^{2} \delta_{x}^{2} / \omega^{2}=\epsilon_{i}^{2}\left(\epsilon^{2}+\delta_{z} \cot \left(\delta_{z}\right)\right)$ are significantly large. This is possible only if respectively $\delta_{z} \approx \delta_{z, m}$ (§4.3.2) or $\delta_{z} \approx \delta_{z, n}$ (§4.3.1). Graphically, figures 4.a, b and d confirm that $\delta_{z}$ varies sharply only in regions where $\delta_{x}^{2}+\delta_{z}^{2} \approx 0$ i.e. in regions where the acoustic gravity wave solutions are close to the (gray) vertical surface $\left(\delta_{x}^{2}+\delta_{z}^{2}=0\right)$.

The study of the poles finally confirms the separation of the branches of the acoustic gravity wave solutions in agreement with the results proved in appendix (A.2). Firstly, a clear separation has now been brought to light in terms of their vertical wave-numbers since the branches are confined to the interval regions locates between the poles: $\left.\delta_{z} \in\right] \delta_{z, m=n}, \delta_{z, n}\left[\right.$ and $\left.\delta_{z} \in\right] \delta_{z, n}, \delta_{z, m=m+1}\left[\right.$ for $n \in \mathbb{N}^{*}$. Secondly, in so far as the not-too-long wave solutions satisfy either $\delta_{z} \approx \delta_{z, m}$ or $\delta_{z} \approx \delta_{z, n}$, the relation (55b) further confirms that the solutions are also well-separated in terms of their angular frequency. Indeed, we have shown that $\delta_{x}^{2}>0$ imposes in this case that $\delta_{z}$ is close to either $\delta_{z, m}$ or $\delta_{z, n}$. Since (i) $\delta_{z, m}$ is a pole of $\omega^{2}$ and thus $\omega^{2}\left(\delta_{z} \approx \delta_{z, m}\right.$ and $\left.\delta_{z, m}<\delta_{z}\right)$ is large and (ii) $\omega^{2}\left(\delta_{z}\right)$ monotonic decreasing and $\omega^{2}\left(\delta_{z, n}\right)=\epsilon_{i}^{2}$, we can conclude and confirm that $\omega^{2}\left(\delta_{z} \approx \delta_{z, m}\right)>>\epsilon_{i}^{2} \geq \omega^{2}\left(\delta_{z} \approx \delta_{z, n}\right.$ and $\left.\delta_{z, n}<\delta_{z}\right)$.

\subsection{Real $\delta_{z}$ : modified internal and acoustic waves}

As shown in $\S(3.3)$ and $\S(4.2)$, upper (acoustic) and lower (gravity) regions of the inner dispersion surface for real $\delta_{z}$ are well-separated: Modified Acoustic Modes (MAM) and Modified Internal Modes (MIM) solutions can thus be studied independently. We additionally showed that as long as $\delta_{z}$ is not close to zero, the acoustic gravity wave solutions remain close to one of the poles. The case of long waves with $\delta_{z} \approx 0$ is discussed in Subsection $\S(4.5)$.

\subsubsection{Development of internal-gravity modes modified by compressibility (MIM)}

Waves can propagate horizontally between the bottom and surface of the ocean as in a wave guide. Internal gravity modes are well-known such examples (Gill, 1982). In §(4.1), graphical inspections of wave solutions confirmed that gravity waves with constrained vertical wave-numbers could be found at the intersection of inner and boundary dispersion surfaces.

We have also shown in $\$ 3.3$ that the root of the inner dispersion relation corresponding to internal gravity waves is well-approximated by $\omega_{i}^{2}(45)$ and in $\S(4.2)$ and $\S(\mathrm{A} .3)$ that $\delta_{z}$ remains close to $\delta_{z, n}$.

In order to refine the approximation and to parameterize this relation in terms of the horizontal wavenumber, we equate the squared angular frequency given by the surface dispersion relation (34b) to $\omega_{-}^{2}$, given by the inner dispersion relation (43). This forms a non-linear equation for $\delta_{z}$ whose solution can be approximated using two passes of a Newton algorithm starting from $\delta_{z}=\delta_{z, n}$. Finally a Taylor development in $\epsilon_{i}, \epsilon_{a}$ leads to:

$$
\delta_{z}\left(\delta_{x}\right)=\delta_{z, \operatorname{mim}}\left(\delta_{x}\right)=\delta_{z, n}\left(1+\frac{\epsilon_{i}^{2}}{\delta_{x}^{2}+\delta_{z, n}^{2}}+\frac{\left(\delta_{x}^{2}-\delta_{z, n}^{2}\right)}{\left(\delta_{x}^{2}+\delta_{z, n}^{2}\right)^{3}} \epsilon_{i}^{4}\right)+\mathrm{O}\left(\epsilon^{6}\right)
$$

A development for the angular frequency $\omega^{2}$ can be obtained by injecting expression (57) of $\delta_{z}$ in the general expression (50) in the unbounded domain case. The usual dispersion relation given in Table 1 writes in dimensional form:

$$
\omega^{2}\left(\delta_{x}\right)=\left.\omega_{i g r}^{2}\right|_{\delta_{z}=\delta_{z, n}}\left(\delta_{x}\right)=\epsilon_{i}^{2} \frac{\delta_{x}^{2}}{\delta_{x}^{2}+\delta_{z, n}^{2}}
$$

Considering only the first order correction to this relation, we can omit all terms of order 3 in $\epsilon^{2}$ in (50) to get:

$$
\omega^{2}\left(\delta_{x}\right)=\omega_{\operatorname{mim}}^{2}\left(\delta_{x}\right)=\underbrace{\epsilon_{i}^{2} \frac{\delta_{x}^{2}}{\delta_{x}^{2}+\delta_{z, n}^{2}}}_{\omega_{i m}^{2}}\left(1-\frac{2 \epsilon_{i}^{2} \delta_{z, n}^{2}}{\left(\delta_{x}^{2}+\delta_{z, n}^{2}\right)^{2}}\right)+\mathrm{O}\left(\epsilon^{6}\right)
$$

Therefore, the main correction to the usual dispersion relation comes from the fact that $\delta_{z}$ is not exactly equal to (but remains close to) $\delta_{z, n}=n \pi$. MIM correspond to the green curve in (figure 3.a). 


\subsubsection{Development of acoustic modes modified by gravity (MAM)}

To study modified acoustic modes at higher angular frequencies, we now use the fact that the root of the inner dispersion relation corresponding to acoustic waves is well-approximated by $\omega_{a}^{2}(46)$ and that $\delta_{z}$ remains close to $\delta_{z, m} \S(4.2)$ and (A.3).

Again, in order to get a more accurate expression of $\delta_{z}$ parameterize by the horizontal wave-number $\delta_{x}$, we equate the (inversed) squared angular frequency given by the surface dispersion relation (34b) to the (inversed) $\omega_{+}^{2}$, given by the inner dispersion relation (43), and perform the nonlinear equation's solution approximation followed by a Taylor development in $\epsilon_{i}, \epsilon_{a}$ to obtain:

$$
\begin{aligned}
\delta_{z}\left(\delta_{x}\right) & =\delta_{z, \operatorname{mam}}\left(\delta_{x}\right)=\delta_{z, m}-\frac{\left(\delta_{x}^{2}-\delta_{z, m}^{2}\right)}{2 \delta_{z, m}\left(\delta_{x}^{2}+\delta_{z, m}^{2}\right)} \epsilon_{a}^{2}+\frac{\epsilon_{i}^{2}}{2 \delta_{z, m}^{2}} \\
& \underbrace{-\frac{\delta_{x}^{6}\left(\epsilon_{a}^{2}-\epsilon_{i}^{2}\right)^{2}+3 \delta_{x}^{2}\left(\epsilon_{a}^{2}-\epsilon_{i}^{2}\right)^{2} \delta_{z, m}^{2}-2 \delta_{x}^{2}\left(5 \epsilon_{a}^{2}-3 \epsilon_{i}^{2}\right) \epsilon^{2} \delta_{z, m}^{4}+2 \epsilon^{4} \delta_{z, m}^{6}}{4\left(\delta_{x}^{2}+\delta_{z, m}^{2}\right)^{3} \delta_{z, m}^{3}}}_{\mathrm{O}\left(\epsilon^{4}\right)}+\mathrm{O}\left(\epsilon^{6}\right)
\end{aligned}
$$

An expansion for the angular frequency $\omega^{2}$ can be obtained by injecting the expression (59) of $\delta_{z}$ in the general expression (53) in the unbounded domain case. The main departure from to the usual acoustic wave angular frequency, given in Table 1 in dimensional form, is given by the second-order development:

$$
\omega^{2}\left(\delta_{x}\right)=\omega_{m a m}^{2}\left(\delta_{x}\right)=\frac{1}{\epsilon_{a}^{2}}\left[\delta_{x}^{2}+\delta_{z, m}^{2}-\frac{\left(\delta_{x}^{2}-\delta_{z, m}^{2}\right)}{\left(\delta_{x}^{2}+\delta_{z, m}^{2}\right)} \epsilon_{a}^{2}+\epsilon_{i}^{2}\right]+\mathrm{O}\left(\epsilon^{2}\right)
$$

Here, stratification has a first-order (in $\epsilon_{i}^{2}$ ) contribution to the modification of the homogeneous case angular frequency. This first-order modification comes from the first-order modification on the vertical wave-number itself (59). However, it is clear that the associated impact is small since $\epsilon_{i}^{2}$ is negligible compared with $\delta_{z, m}^{2}$ in (59) and (60), because $\delta_{z, m} \geq \pi / 2$. A similar conclusion was drawn in (Smith, 2015). MAM correspond to the red curves in (3.a).

\subsection{Purely imaginary $\delta_{z}$ : modified surface acoustic-gravity waves (MSW)}

"Surface waves" generally refer to wave propagating horizontally as anomalies of the ocean free-surface (Gill, 1982). In the vertical direction, these surface wave anomalies are "evanescent", meaning that, with the notation chosen in the present study, the vertical wave-number $\delta_{z}$ is a purely imaginary complex number. A Modified Surface Wave (MSW) defined by its triplet $\left(\delta_{x}, \delta_{z}, \omega\right)$ must satisfy both the inner (39a) and boundary (39b) dispersion relations for $\delta_{z}=i \delta_{z, i}$.

Homogeneous \& incompressible ocean. In an homogeneous and incompressible ocean $\left(\epsilon_{i}=\epsilon_{a}=0\right.$, figure 2), the inner dispersion relation (39a) implies $\delta_{z, i}=\delta_{x}$. This equality is often postulated in textbooks to reduce the number of variables. Vertical polarization relations are then functions of $\delta_{x}$ only (Gill, 1982) and, as a consequence, the only remaining dispersion relation is the boundary dispersion relation (39b) for purely imaginary vertical wave-number $\left(\delta_{z}=i \delta_{x}\right)$, or its approximation $\omega^{2}=\delta_{x} \tanh \delta_{x}$. In this case, $\delta_{z, i}$ is reduced to a vertical length-scale of energy decay with increasing distance from the surface; for very long waves $\left(\delta_{x} \gg 1\right)$, the surface wave (LSW) is approximately depth-independent (table 1).

Dispersion relation. In the more general case (non homogeneous and compressible ocean), we prove in Appendix (A.4) the existence of solutions to (39a, 39b). When the parameters $\epsilon_{i}$ and $\epsilon_{a}$ are small, we additionally prove in Appendix (A.5) that surface acoustic-gravity waves have approximately similar horizontal and vertical wave-numbers $\left(\delta_{x} \approx \delta_{z, i}\right)$. 
This crude assumption $\delta_{x} \approx \delta_{z, i}$ is sufficiently accurate to recover usual swell-like approximations (Table 1 ), i.e., for sufficiently large $\delta_{x}$ (or $\delta_{z}$ ). However a more accurate expression is given by:

$$
\delta_{z, i}^{2}\left(\delta_{x}\right)=\delta_{x}^{2}-\delta_{x}\left(\frac{\epsilon_{i}^{2}}{\tanh \left(\delta_{x}\right)}+\epsilon_{a}^{2} \tanh \left(\delta_{x}\right)\right)+\mathrm{O}\left(\epsilon^{4}\right) .
$$

In order to obtain (61), we introduce the angular frequency given by the boundary dispersion relation in the inner dispersion relation (39a) and solve the resulting nonlinear equation by performing one pass of a Newton algorithm. Note that the problem is formulated in terms of $\delta_{z, i}^{2}\left(\delta_{x}\right)$ since it can be shown (by looking at the error estimate of the Newton algorithm) that a formulation in terms of $\delta_{z, i}\left(\delta_{x}\right)$ is not accurate when $\delta_{x}$ is relatively small. Note also that (61) requires $\delta_{x} \geq \epsilon_{i}$ for $\delta_{z, i}^{2}$ to be positive. This is consistent with the fact that $\delta_{x}$ must be greater than $\delta_{x, 0}\left(\approx \epsilon_{i}\right)$ defined in (37), as shown in Appendix (A.4). However (61) does not allow us to recover the exact value $\delta_{x, 0}$ of $\delta_{x}$ that cancels $\delta_{z, i}$, due to the first-order only approximation in terms of $\epsilon^{2}$ ). The case of long surface waves with $\delta_{z, i}$ close to zero will be treated separately in $\S 4.5$.

The angular frequency $\omega^{2}\left(\delta_{x}\right)$ associated to the vertical wave-number $\delta_{z, i}^{2}\left(\delta_{x}\right)$ given by (61) is well approximated by:

$$
\omega^{2}\left(\delta_{x}\right)=\delta_{x} \tanh \left(\delta_{x}\right)\left[1-\frac{1}{2}\left(\frac{\epsilon_{i}^{2}}{\sinh ^{2}\left(\delta_{x}\right)}+\frac{\epsilon_{a}^{2}}{\cosh ^{2}\left(\delta_{x}\right)}-\frac{\epsilon_{i}^{2}}{\delta_{x} \sinh \left(\delta_{x}\right) \cosh \left(\delta_{x}\right)}\right)\right]+\mathrm{O}\left(\epsilon^{4}\right)
$$

For very short waves $\left(\delta_{x} \gg 1\right)$, these relations simplify to:

$$
\delta_{z, i} \approx \delta_{x}-\epsilon^{2}, \quad \omega^{2} \approx \delta_{x}
$$

and we recover the angular frequency of short non hydrostatic waves $\omega^{2}=\delta_{x}$ (or $\Omega=\sqrt{g k_{x}}$ in dimensional form) with a slightly modified vertical wave-number. MSW correspond to the blue curve in (3.a).

Marginally stable surface gravity waves. We proved in $\S(3.3)$ that the ratio $R^{2}\left(\delta_{x}, \delta_{z}\right)$ is maximum and equal to $1 / 4$ only when $\delta_{z}$ is pure imaginary. The relations (47a) and (47b) are simultaneously satisfied (gray curve in figure 2.d). Any triplet $\left(\delta_{x}, \delta_{z}, \omega\right)$ satisfying these two relations is an acceptable wave solution only if it also satisfies the boundary dispersion relation (39b). As a consequence, $\delta_{x}(\omega)$ and $\delta_{z, i}(\omega)$ can be obtained combining (47b) and (39b) (both relations are recalled below), then (47a) leads to the non-linear equation for $\omega$ given by (63a):

$$
\begin{aligned}
& \delta_{x}=\frac{\epsilon_{a}}{\epsilon_{i}} \omega^{2} \\
& \omega^{2}=\frac{\delta_{x}^{2} \tanh \left(\delta_{z, i}\right)}{\delta_{z, i}+\epsilon^{2} \tanh \left(\delta_{z, i}\right)} \\
& \delta_{x}^{2}(\omega)-\delta_{z, i}^{2}(\omega)-\epsilon_{i}^{2} \frac{\delta_{x}^{2}(\omega)}{\omega^{2}}-\epsilon_{a}^{2} \omega^{2}+\epsilon^{4}=0
\end{aligned}
$$

For the stratification and acoustic parameters $\epsilon_{i}$ and $\epsilon_{a}$ given in Table (2), Equation (63a) has a unique solution for $\omega_{*} \approx 0.154$. This wave solution (red point in figure 5$)$ is marginally stable $\left(\omega_{+}=\omega_{-}\right.$and $\left.R^{2}\left(\delta_{x}, \delta_{z}\right)=1 / 4\right)$. Further investigations of this peculiar region of surface-wave phase-space must in particular take into account the consequences of earth rotation and wave non-linearity.

\subsection{Long waves}

We now prove the existence of two sub-branches of long wave solutions: mode-0 Modified Internal Modes (MIM-0) with horizontal wave-numbers in the range $\delta_{x} \in\left[0, \delta_{x, 0}\right]$ and Long Modified Surface Waves (LMSW) in the range $\delta_{x} \in\left[\delta_{x, 0},+\infty[\right.$. 


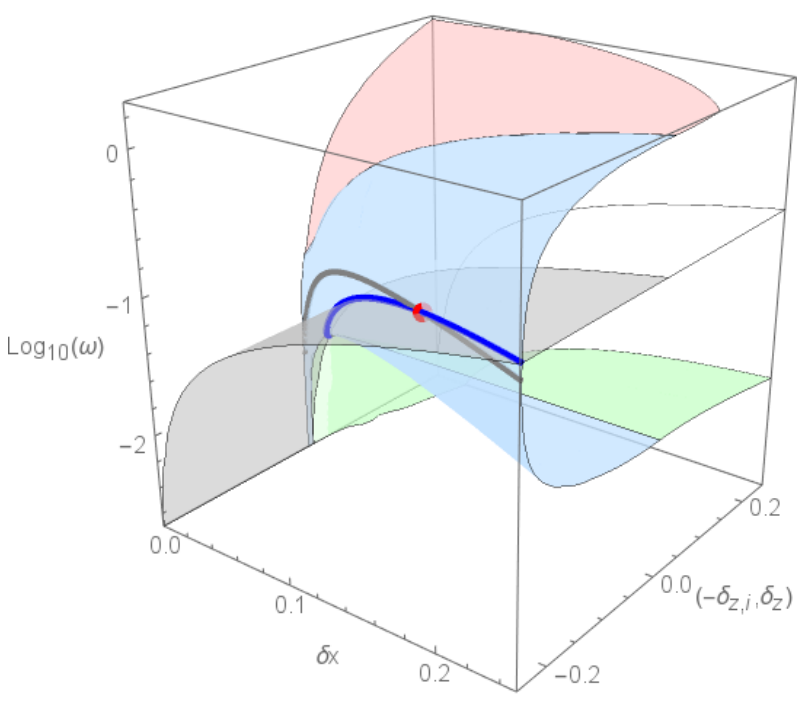

Figure 5: inner and boundary dispersion surfaces in $\left(\delta_{x}, \delta_{z}, \omega\right)$ space and wave solutions for long waves. Colors: (light red) acoustic wave region ( $\delta_{z}^{2} \geq 0 \mathcal{E} \omega$ large), (light blue) surface wave region $\left(\delta_{z}^{2} \geq 0 \mathcal{E}\right.$ $\omega$ small), (light green) internal wave region $\left(\delta_{z}^{2}<0\right)$, (white) boundary dispersion surface for $\delta_{z} \in \mathbb{R}$, (light gray) boundary dispersion surface for $\delta_{z} \in \mathbb{R}$. Blue curve: Modified Surface Waves (MSW). Gray curve: triplets $\left(\delta_{x}, \delta_{z}, \omega\right)$ satisfying (47b) and (47a). Red Point: marginally stable MSW $\S(4.5)$.

Dispersion relations for long waves. Here, we perform specific developments for long waves where the vertical profile is almost depth-independent $\left|\delta_{z}\right| \approx 0, \delta_{z}$ being either real or purely imaginary (figure $3 \mathrm{~b})$. This corresponds to the long-wave part of the acoustic-gravity branch located in the region $\delta_{z}^{2} \in$ ] $-\infty, \delta_{z, m=1}^{2}$ ] of figure $4 \mathrm{a}$.

Inserting the boundary dispersion relation (34b) into the inner dispersion relation (34a) and making a second-order Taylor expansion in $\delta_{z}$ leads to:

$$
\delta_{z}^{2}\left(\delta_{x}\right)=\delta_{z, \text { long }}^{2}\left(\delta_{x}\right)=\left(\delta_{x, 0}^{2}-\delta_{x}^{2}\right) \frac{1+\epsilon_{i}^{2} / 3-\epsilon_{a}^{2}}{\left(1+\epsilon_{i}^{2} / 3\right)\left(1+\epsilon_{i}^{2} / 3\right)-\delta_{x}^{2} \epsilon_{a}^{2} / 3}+\mathrm{O}\left(\delta_{z}^{4}\right)
$$

$\delta_{x, 0}$ is the value of $\delta_{x}$ for which $\delta_{z}=0$ is a solution of the inner and boundary dispersion relations ( $(2.6)$. At first order in $\epsilon^{2}, \delta_{x, 0}$ is equal to $\epsilon_{i}$. The corresponding angular frequency can be obtained by inserting the approximation of the vertical wave-number given by (64) into the boundary relation dispersion (34b). An approximation at second order in $\delta_{x}^{2}$ and $\epsilon^{2}$ is:

$$
\omega^{2}\left(\delta_{x}\right)=\omega_{\text {long }}^{2}\left(\delta_{x}\right)=\delta_{x}^{2}\left(1-\frac{1}{6}\left(\epsilon_{i}^{2}+3 \epsilon_{a}^{2}\right)+\frac{1}{3} \epsilon_{a}^{2} \epsilon^{2}+\frac{1}{45} \epsilon_{i}^{4}+\mathrm{O}\left(\epsilon^{6}\right)\right)+\mathrm{O}\left(\delta_{x}\right)^{4} .
$$

We also define:

$$
\delta_{z, 0}^{2}\left(\delta_{x}=0\right)=\frac{\delta_{x, 0}^{2}\left(1+\epsilon_{i}^{2} / 3-\epsilon_{a}^{2}\right)}{\left(1+\epsilon_{i}^{2} / 3\right)\left(1+\epsilon_{i}^{2} / 3\right)-\delta_{x}^{2} \epsilon_{a}^{2} / 3}+\mathrm{O}\left(\delta_{z}^{4}\right)
$$

Figure (6) shows the evolution of $\delta_{z}^{2},\left|\delta_{z}\right|$ and $\omega$ as functions of $\delta_{x}$ for long waves (6a, b and d) and for short and intermediate waves (6c).

$\delta_{x} \leq \delta_{x, 0}$. For $\delta_{x} \leq \delta_{x, 0} \approx \epsilon_{i}$, the vertical wave-number $\delta_{z}$ given by (64) is real. This implies that $\omega^{2} \approx \delta_{x}^{2} \leq \epsilon_{i} \delta_{x} \leq \frac{\epsilon_{i}}{\epsilon_{a}} \delta_{x}$ (since $\epsilon_{a}<1$ ) and, thus, using the bounds on $\omega_{-}^{2}, \omega_{+}^{2}$ given by (44), that these waves are always issued from the internal-wave region of the inner dispersion surface $\left(\omega_{-}^{2}\right)$, not from the acoustic-wave region $\left(\omega_{+}^{2}\right)$. In other words, there is no intersection between the boundary dispersion 


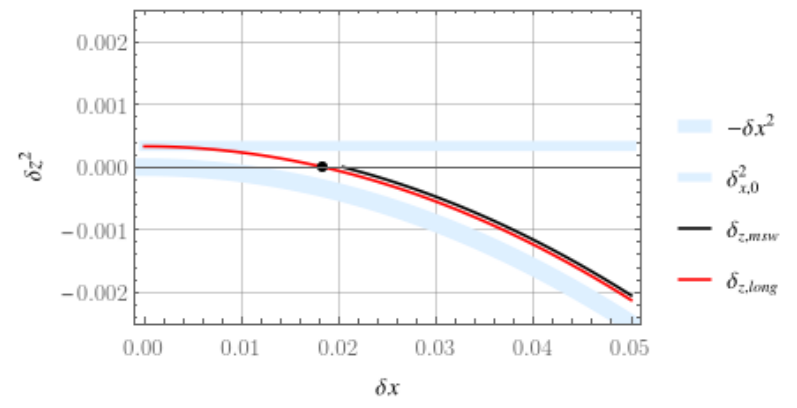

(a)

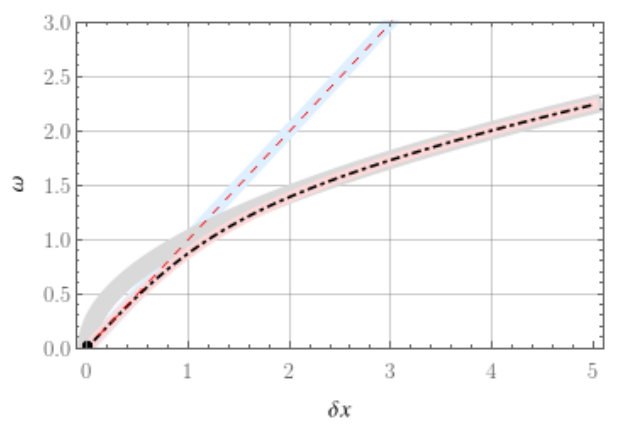

(c)

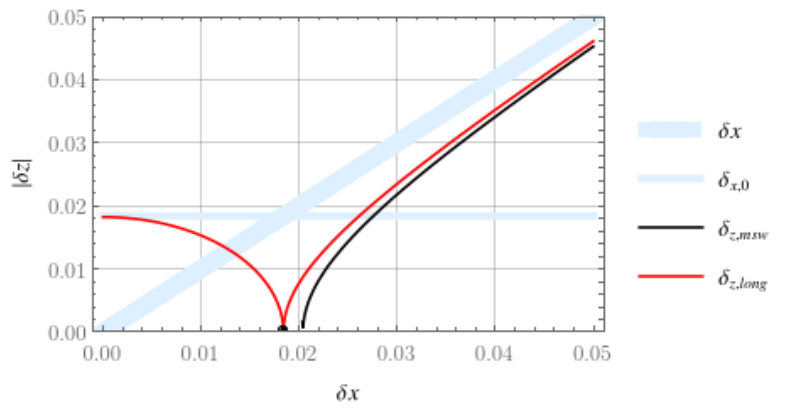

(b)

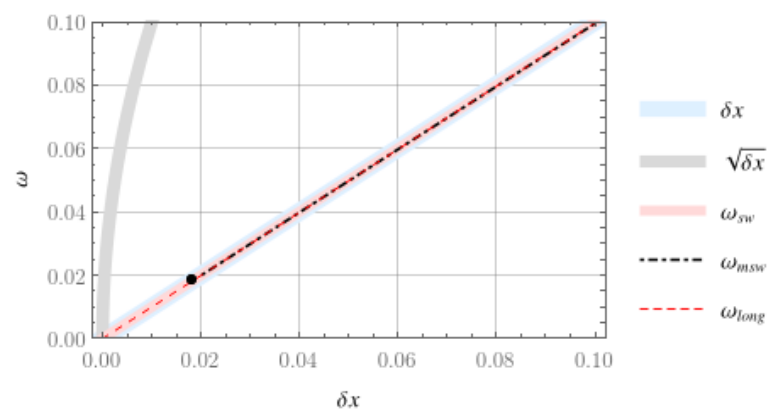

(d)

Figure 6: dispersion curves for (intermediate) and long wave-lengths $\delta_{z}$. Black point: depth-independent surface gravity wave. $\$(2.6)$.

(a) square modulus of the vertical wave-number $\delta_{z}^{2}$ as a function of the horizontal wave-number $\delta_{x}$. The exact solution can be numerically computed (not shown) and is visually not distinguishable from the blue curve (accurate approximation). The vertical wave-number of an incompressible and homogeneous ocean would follow the straight line $\delta_{z}=\delta_{x}$.

(b) modulus of the vertical wave-number $\left|\delta_{z}\right|$ as a function of the horizontal wave-number $\delta_{x}$. The exact solution can be numerically computed (not shown) and is visually not distinguishable from the blue curve (accurate approximation). The vertical wave-number of an incompressible and homogeneous ocean would follow the straight line $\delta_{z}=\delta_{x}$.

(c) Angular frequency $\omega$ as a function of $\delta_{x}$ the horizontal wave-number for different approximations (short, intermediate and long waves).

(d) Angular frequency $\omega$ as a function of $\delta_{x}$ but in the vicinity of the origin.

relation and the (real) acoustic-wave region for $\delta_{z} \in \mathbb{R}$ close to zero for the chosen boundary conditions . This is consistent with the fact that (acoustic) Lamb waves can only be solutions for different surface or bottom boundary conditions in $\S(2.6)$.

Dukowicz (2013) notes that this wave solution, like any higher internal modes, has its angular frequency $\omega^{2}$ approximately bounded by $\epsilon_{i}^{2}$ (i.e. $\Omega \leq N$ ), since $\omega^{2} \approx \delta_{x}^{2} \leq \epsilon_{i}^{2}$. We however avoid to refer to this $\mathrm{n}=0$ internal mode (MIM0) as a barotropic wave. Indeed if $\epsilon_{i}=\epsilon_{a}=0$, i.e. if stratification and compressibility vanish in this sub-branch disappears. Since $\delta_{z} \in \mathbb{R}$, MIM0 propagates in the vertical direction but its vertical wave-number remains small even in strongly-stratified regions (large values of $\epsilon_{i}$, see table (6)).

$\boldsymbol{\delta}_{\boldsymbol{x}} \geq \boldsymbol{\delta}_{\boldsymbol{x}, \mathbf{0}}$. For $\delta_{x} \geq \delta_{x, 0}$ (but still small), $\delta_{z}$ given by (64) is purely imaginary. These long waves originate from the surface-wave branch MSW and will here be named LMSW. When $\delta_{x}$ increases, $\delta_{z}$,long (64) converges to $\delta_{z, l m s w}(61)$, which is a better approximation for medium and short surface waves. Figure (6.a) shows $\delta_{z}^{2}$ as a function of $\delta_{x}$ (for $\delta_{x} \leq 2 \epsilon_{i}$ ). The two approximations given by (64), accurate for long waves, and (61), accurate for medium/short waves, are plotted. On Figure (6.b), the modulus of 
the vertical wave-number is shown on the same interval for $\delta_{x}$ (for $\delta_{x} \leq 2 \epsilon_{i}$ ).

Figure (6.c and d) present the angular frequency $\omega$ as a function of the horizontal wave-number $\delta_{x}$ according to different approximations for small, intermediate and long horizontal wave-numbers. $\omega_{\text {long }}$ and $\omega_{m s w}$ are computed by inserting the corresponding approximations (64) and (61) of $\delta_{z}$ in the boundary dispersion relation (34b). $\omega=\delta_{x}$ (resp. $\omega=\sqrt{\delta_{x}}$ ) represents the classical long shallow-water waves (resp. short non-hydrostatic surface waves) approximation. $\omega=\omega_{s w}=\sqrt{\delta_{x} \tanh \left(\delta_{x}\right)}$ is the angular frequency of an homogeneous and incompressible (non hydrostatic) ocean. Unsurprisingly, this last approximation is accurate over the full range of horizontal wave-numbers, even for the set of compressible stratified equations. Indeed, even if for long waves, $\delta_{z}$ is not directly linked to $\delta_{x}$ (in particular $\delta_{z}$ does not vanish for $\delta_{x} \approx 0$ ), $\delta_{z}$ remains small (less than $\epsilon_{i}$ ) and thus $\delta_{z} / \tan \left(\delta_{z}\right)$ is close to 1 .

Interestingly, and contrary to conclusions in (Dukowicz, 2013), the MIM-0 angular frequency cannot saturate at the buoyancy angular frequency for increasing horizontal wave-number $\delta_{x}$ but transforms into a vertically evanescent surface wave, whereas its vertical wave-number switches from real to purely imaginary.

Hydrostaticity and asymptotic behaviour. Surface edge waves are consequently either MIM0 Modes $\left(\delta_{z} \in \mathbb{R}\right.$ and $\left.\delta_{x}<\delta_{x, 0}\right)$ or Modified Surface Waves (MSW, $\delta_{z} \in i \mathbb{R}$ and $\left.\delta_{x}>\delta_{x, 0}\right)$. For $\delta_{x}=\delta_{x, 0}$, a depthindependent gravity surface wave studied in $\S(2.6)$ separates these two sub-branches. In the long wave approximation, the "long wave solution" (61) is an accurate approximation of both MIM0 and LMSW. We now further prove that in the long-wave, low-angular frequency limit:

- for $\delta_{x}<\delta_{x, 0}$, the MIM0 is (quasi-) hydrostatic and converges towards the $\sqrt{g H}$-phase-velocity shallow-waver solution (SHW) when stratification loosens (i.e. when $\epsilon_{i} \rightarrow 0$ )

- for $\delta_{x}>\delta_{x, 0}$, the LMSW solution remains non-hydrostatic and converges towards the other $\sqrt{g H}$ phase-velocity long-swell solution (LSW) when stratification loosens (i.e. when $\epsilon_{i} \rightarrow 0$ ).

The SHW and LSW asymptotic solutions are recalled in Table 1 and discussed in $\S(2.6)$.

Using the approximate solution for long waves (64) and for LMSW (61), we can compute their respective aspect-ratio:

$$
\begin{aligned}
& r_{\text {long }}^{2}\left(\delta_{x}\right)=\frac{\delta_{x}^{2}}{\delta_{z, \text { long }}^{2}}=\delta_{x}^{2} \frac{1+\epsilon_{i}^{2} / 3-\epsilon_{a}^{2}}{\left(1+\epsilon_{i}^{2} / 3\right)\left(1+\epsilon_{i}^{2} / 3\right)-\delta_{x}^{2} \epsilon_{a}^{2} / 3}+\mathrm{O}\left(\delta_{z}^{4}\right) \\
& r_{m s w}^{2}\left(\delta_{x}\right)=\frac{\delta_{x}^{2}}{\delta_{z, \text { msw }}^{2}}=\frac{\delta_{x}}{\epsilon_{a}^{2} \tanh ^{2}\left(\delta_{x}\right)-\delta_{x} \tanh \left(\delta_{x}\right)+\epsilon_{i}^{2}}
\end{aligned}
$$

Figure (7) shows the evolutions of these aspect ratios as functions of the horizontal wave-number. A study of the variations of $r_{l o n g}$ shows that this ratio increases monotonically from 0 to infinity when $\delta_{x}$ varies from 0 to $\delta_{x, 0}$ and decreases monotonically from infinity to 1 when $\delta_{x}$ varies from $\delta_{x, 0}$ to infinity. In this latter range, $r_{m s w}$ remains close to $r_{\text {long. }}$. Except in the vicinity of $\delta_{x, 0}$, the aspect ratio $r_{\text {long }}$ remains lower than 1 and it decreases monotonically to 0 when $\delta_{x}$ approaches 0 . In this (very) long-wave range, the MIM-0 solution is thus hydrostatic and the dispersion relations (64) and (62) are approximately given by:

$$
\delta_{z}^{2}=\delta_{z, 0}^{2} \approx \epsilon_{i}^{2} \quad \& \quad \omega^{2} \approx \delta_{x}^{2}
$$

This solution is also the long-wave approximation of the hydrostatic wave solution (Appendix B).

The second branch is a monotonous function for $\delta_{x} \in\left[\delta_{x, 0},+\infty\right.$ [ and it decreases to 1 when $\delta_{x} \rightarrow+\infty$. As a consequence, it remains superior to 1 in this range of wave-numbers, the corresponding wave solution is non-hydrostatic and the dispersion relations (64) and (62) are approximately given by:

$$
\delta_{z, i}^{2} \approx \delta_{x}^{2}-\epsilon_{i}^{2} \quad \& \quad \omega^{2} \approx \delta_{x}^{2}
$$

This solution is also the long-wave approximation of the non-hydrostatic wave solution (Appendix B). 


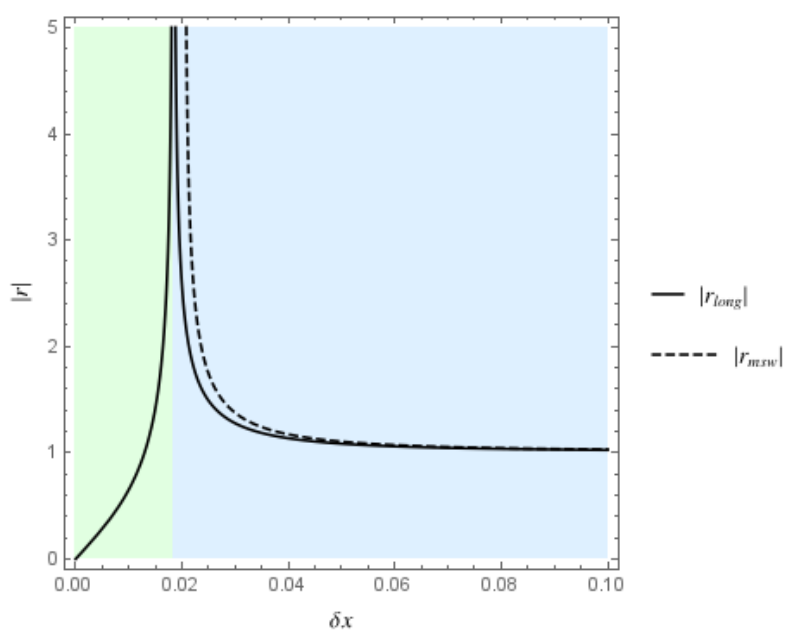

Figure 7: modulus of the wave aspect ratios $r_{\text {mim } 0}$ (black-solid curve) and $r_{\text {lmsw }}$ (black, dashed curve). Colors: (light-green) internal wave region $\left(\delta_{z} \in \mathbb{R}\right)$, (light-blue) surface wave $\left(\delta_{z} \in i \mathbb{R}\right)$

\subsection{Summary: waves solutions in a bounded ocean}

Unlike in an unbounded ocean, acoustic-gravity wave solutions in a bounded, free-surface ocean must satisfy both the inner and boundary dispersion relations. This induces the appearance of poles in these dispersion relations for $\delta_{z}=\frac{\pi}{2}+(m-1) \pi(\mathrm{MAM})$ and for $\delta_{z}=n \pi(\mathrm{MIM})$ with $(m, n) \in \mathbb{N}^{*} x \mathbb{N}^{+}$. For $\delta_{z} \in i \mathbb{R}$, the boundary dispersion relation does not exhibit any pole.

A stratified ocean can propagate two types of surface edge waves:

- for medium and short wave-lengths $\left(\delta_{x}>\delta_{x, 0}\right)$, MSW are non-hydrostatic $\left(r_{l m s w}>1\right)$, evanescent $\left(\delta_{z, s w} \in i \mathbb{R}\right)$ and converge towards the $\sqrt{g H}$-phase-velocity, depth-dependent $\left(\delta_{x}=\delta_{z, i}\right)$ wave solution (LSW) when both $\epsilon_{i} \rightarrow 0$ and $\delta_{x} \rightarrow \delta_{x, 0}$. Vertical variations of pressure are nonhydrostatic and in a homogeneous ocean, LMSW solutions remain depth-dependent $\left(\delta_{x}=\delta_{z, i} \neq 0\right)$.

- for longer waves $\left(\delta_{x}<\delta_{x, 0}\right)$, MIM0 are quasi-hydrostatic $\left(r_{\operatorname{mim} 0}<1\right.$ if $\delta_{x}$ is not in the neighbourhood of $\left.\delta_{x, 0}\right)$, vertically propagating $\left(\delta_{z} \in \mathbb{R}\right)$ and converges towards the shallow-water $\sqrt{g H}$-phasevelocity solution (SHW) when both $\epsilon_{i} \rightarrow 0$ and $\delta_{x} \rightarrow 0$. In this case, vertical variations of pressure are hydrostatic and LMIW solutions are depth-independent $\left(\delta_{z}=0\right)$. When the stratification loosens $\left(\epsilon_{i} \rightarrow 0\right)$, the range of validity of MIM0 solution $\left(0<\delta_{x}<\delta_{x, 0} \approx \epsilon_{i}\right)$ shrinks to zero.

The angular frequency of acoustic wave solutions (modified by gravity) remains larger than $\omega_{c, a}=\epsilon^{2} / \epsilon_{a}$ whereas the angular frequency of gravity waves (modified by compressibility) remains smaller than $\omega_{c, i}=\epsilon_{i}(\S 3.3)$.

Table 5 summarizes the main approximations for the different type of waves (acoustic, gravity and surface waves/modes) given in this paper. These approximations are indicated in their dimensional form. For sake of readability, some of the relations given in Table 5 are lower order versions of our derivations. They generally stem from first-order correction terms, compared with usual dispersion relations introduced in Table 1. In that case, a red link to the higher approximation is given.

Table (6) gives orders of magnitude of various characteristics of these acoustic gravity waves for the reference set of $\left(\epsilon_{a}, \epsilon_{i}\right)$ parameters (table 2$)$ and for a shallow or a strongly stratified ocean.

For the 4000m-deep reference ocean, the horizontal length scale associated with the transformation of the MSW into the long MIM $\left(\lambda_{x, l m s w}=2 \pi /\left(\delta_{x, l m s w} / 4000\right)\right)$ reaches $1367 \mathrm{~km}$ against $62 \mathrm{~km}$ for the same $10 \mathrm{~m}$-deep ocean. When $\delta_{x}$ decreases below $\delta_{x, l m s w}, \delta_{z}$ increases monotonically to a maximum value $\delta_{z, \text { lmsw }}\left(\delta_{x}=0\right)$. This vertical length scale reaches $1379 \mathrm{~km}$ for the $4000 \mathrm{~m}$-deep reference ocean and 62 $\mathrm{km}$ for the same $10 \mathrm{~m}$-deep ocean. A rule can be formulated as: the longer the horizontal length scale 


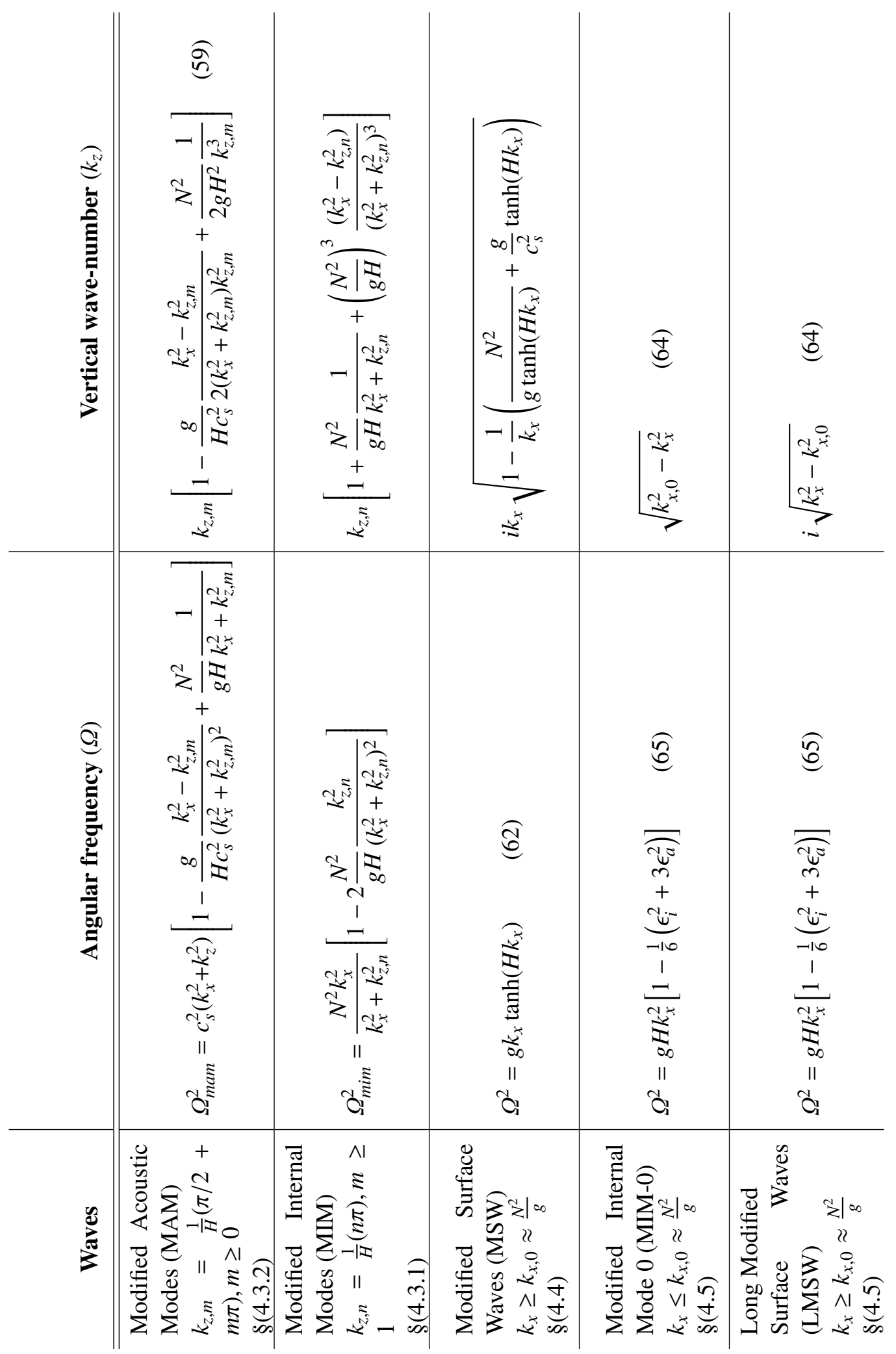

Table 5: Compressibility and stratification induced modifications to the usual dispersion relations given in table $1 . \Omega$ is wave angular frequency, $k_{x}$ and $k_{z}$ are the horizontal and vertical wave-numbers, $g$ is the acceleration of gravity, $N$ a reference Brunt-Väisälä angular frequency and $c_{s}$ the speed of sound. $D_{0}$ is the (incompressible) background density vertical scale and is given by $1 / D_{0}=N^{2} / g+g / c_{s}^{2}$

of the oscillation, the shorter its vertical length scale; and the weaker the stratification (vanishing $\epsilon_{i}$ ) the 


\begin{tabular}{l|l|l|l|l} 
& \multicolumn{1}{|c|}{ Notation } & Reference & $\mathbf{1 0 - m - d e e p}$ & $N=510^{-3} \mathrm{~s}^{-1}$ \\
\hline \hline Parameters & $\epsilon_{a}$ & 0.13 & $6.610^{-3}$ & 0.13 \\
& $\epsilon_{i}$ & $2.010^{-2}$ & $1.010^{-3}$ & 0.1 \\
\hline Acoustic cut-off & $2 \pi \sqrt{H / g} / \omega_{c, a}$ & $31.3 \mathrm{mn}$ & $31.3 \mathrm{mn}$ & $20 \mathrm{mn}$ \\
\hline Internal cut-off & $2 \pi \sqrt{H / g} / \omega_{c, i}$ & $1.7 \mathrm{~h}$ & $1.7 \mathrm{~h}$ & $21 \mathrm{mn}$ \\
\hline Depth-independent surface & $2 \pi H / \delta_{x, 0}$ & $1367 \mathrm{~km}$ & $62 \mathrm{~km}$ & $247 \mathrm{~km}$ \\
gravity wave & $2 \pi \sqrt{H / g} / \omega_{0}$ & $1.9 \mathrm{~h}$ & $1.7 \mathrm{~h}$ & $21 \mathrm{mn}$ \\
\hline MIW, $\delta_{z}(0)$ & $2 \pi H / \delta_{z, 0}$ & $1380 \mathrm{~km}$ & $62 \mathrm{~km}$ & $250 \mathrm{~km}$ \\
& $2 \pi \sqrt{H / g} / \omega_{z, 0}$ & $\infty$ & $\infty$ & $\infty$ \\
\hline MSW, marginally-stable & $2 \pi H / \delta_{x, *}$ & $162 \mathrm{~km}$ & $408 \mathrm{~m}$ & $25 \mathrm{~km}$ \\
wave & $2 \pi H / \delta_{z, *}$ & $164 \mathrm{~km}$ & $408 \mathrm{~m}$ & $25 \mathrm{~km}$ \\
& $2 \pi \sqrt{H / g} / \omega_{*}$ & $13.7 \mathrm{mn}$ & $41.6 \mathrm{~s}$ & $2.4 \mathrm{mn}$
\end{tabular}

Table 6: orders of magnitude of various scales. Notations refer to non-dimensional variables whereas orders of magnitude are given for dimensional quantities. Parameters for the "Reference" ocean are given in Table (2). "10-m-deep" ocean is a 10-m-deep Reference ocean whereas a " $N=510^{-3} s "$ ocean is a reference ocean with $N=510^{-3} s$.

longer its horizontal length-scale $\lambda_{x, l m s w}$. This long MIM solution is a low-angular frequency oscillation of the ocean due to gravity and associated with ocean stratification. It disappears when stratification vanishes and the ocean becomes an homogeneous layer of water. It does persist in an incompressible ocean but is slightly modified by compressibility.

\section{Discussion, conclusion}

An Eulerian, analytical model for acoustic gravity waves propagating in a compressible, stratified, free-surface, non-rotating ocean has been derived and investigated with several objectives: recover acoustic waves and modes in a unified dispersion model, describe analytically the modifications of the characteristics of these waves and modes for small changes in compressibility or stratification and, finally, anticipate the impact of compressibility in the new generation of non-hydrostatic, compressible, ocean models (Auclair et al., 2018).

An original investigation (at least to the authors' knowledge) based on 3D graphics in $\left(\delta_{x}, \delta_{z}, \omega\right)$ phasespace has been carried out to support and illustrate the analytical developments. Well-known acoustic and internal modes (MAM and MIM) have in particular been recovered and have been associated to the infinite number of discrete poles of the inner and boundary dispersion relations. A least two peculiar regions of the phase space have been investigated in more details. Firstly, the long acoustic gravity wave branch has indeed been decomposed in two sub-branches: one associated to a modified mode- 0 internal mode (MIM0) with a real vertical wave-number, the other to a modified long surface gravity wave (LMSW) with pure imaginary vertical wave-number. Secondly, modified surface gravity waves (MSW) have been shown to be marginally stable for intermediate wave-numbers in a region of phase space where compressibility and stratification induced effects approximately compensate.

Analytical solutions are given in dimensional form in Appendix (C), such solutions can conveniently be used to validate non-hydrostatic, compressible, free-surface ocean models (Auclair et al., 2018).

\section{Acknowledgements}

We greatly thank the computer service of the Laboratoire d'Aérologie for NUWA, our laboratory cluster. The present study has been supported by French-Navy SHOM contract GIBRALTAR, by LEFE contract GEPETO, by ANR grant SOLAB, by IDEX Chair of attractivity TEASAO and by H2020 contract IMMERSE. 


\section{References}

Apel, J. R. (1987). Principles of Ocean Physics. Number v. 38 in International Geophysics Series. Academic Press, London ; Orlando.

Auclair, F., Bordois, L., Dossmann, Y., Duhaut, T., Paci, A., Ulses, C., and Nguyen, C. (2018). A nonhydrostatic non-Boussinesq algorithm for free-surface ocean modelling. Ocean Modelling, 132:12-29.

Dukowicz, J. K. (2013). Evaluation of Various Approximations in Atmosphere and Ocean Modeling Based on an Exact Treatment of Gravity Wave Dispersion. Monthly Weather Review, 141(12):44874506.

Eckart, C. (1960). HYDRODYNAMICS OF OCEANS AND ATMOSPHERES. page 293.

Gill, A. E. (1982). Atmosphere-Ocean Dynamics. Academic Press Inc, San Diego.

Jensen, F. B., Kuperman, W. A., Porter, M. B., and Schmidt, H. (2011). Computational Ocean Acoustics. Springer New York, New York, NY.

LeBlond, P. H. and Mysak, L. A. (1981). Waves in the Ocean. Elsevier.

Lighthill, M. J. (1967). Waves in Fluids. pages 267-293.

Pedlosky, J. (2013). Geophysical Fluid Dynamics. Springer Science \& Business Media.

Smith, J. A. (2015). Revisiting Oceanic Acoustic Gravity Surface Waves. Journal of Physical Oceanography, 45(12):2953-2958.

Tolstoy, I. (1963). The Theory of Waves in Stratified Fluids Including the Effects of Gravity and Rotation. Reviews of Modern Physics, 35(1):207-230. 


\section{Appendices}

\section{A Mathematical demonstrations}

\section{A.1 $\delta_{z}$ real or purely imaginary}

In this section, we prove that under the condition of smallness of parameters $\epsilon_{i}$ and $\epsilon_{a}, \delta_{z}$ is either real or pure imaginary and thus that the angular frequency $\omega$ is real.

Polarization function in s-coordinates. The eigenvalue problem (27) and the surface boundary condition (27b) are rewritten in non-dimensional form as:

$$
\begin{aligned}
G^{\prime \prime}(s)+\delta_{z}^{2} G(s) & =0 \\
G(1) & =1 \\
G(0) & =0
\end{aligned}
$$

$$
G^{\prime}(1)+\left(\frac{\epsilon_{i}^{2}+\epsilon_{a}^{2}}{2}-\frac{\delta_{x}^{2}}{\omega^{2}}\right) G(1)=0
$$

where $s=\frac{z+H}{H}, G(s(z))=F(z)$ and $\epsilon_{i}^{2}=\frac{N^{2} H}{g}, \epsilon_{a}^{2}=\frac{g H}{c_{s}^{2}}, \delta_{x}=k_{x} H, \delta_{z}=k_{z} H, \omega=\Omega \sqrt{\frac{H}{g}}$.

Energy equation. $\delta_{z}$ is linked to $\delta_{x}$ and $\omega$ by the inner dispersion relation

$$
\delta_{z}^{2}=\left(\delta_{x}^{2} \frac{\epsilon_{i}^{2}-\omega^{2}}{\omega^{2}}+\epsilon_{a}^{2} \omega^{2}-\frac{\left(\epsilon_{i}^{2}+\epsilon_{a}^{2}\right)^{2}}{4}\right)
$$

Multiplying (A.1) by $\overline{G(s)}$ and integrating over $[0,1]$ we get:

$$
\int_{0}^{1} G^{\prime \prime}(s) \overline{G(s)} \mathrm{d} s+\delta_{z}^{2} \int_{0}^{1}|G(s)|^{2} \mathrm{~d} s=0
$$

Integration by parts leads to:

$$
-\int_{0}^{1}\left|G^{\prime}(s)\right|^{2} \mathrm{~d} s+\delta_{z}^{2} \int_{0}^{1}|G(s)|^{2} \mathrm{~d} s+G^{\prime}(1) \overline{G(1)}-G^{\prime}(0) \overline{G(0)}=0
$$

and using (A.2), (A.3), (A.4)

$$
\delta_{z}^{2} \int_{0}^{1}|G(s)|^{2} \mathrm{~d} s+\left(\frac{\delta_{x}^{2}}{\omega^{2}}-\frac{\epsilon_{i}^{2}+\epsilon_{a}^{2}}{2}\right)=\int_{0}^{1}\left|G^{\prime}(s)\right|^{2} \mathrm{~d} s
$$

Using the Poincaré inequality $\int_{0}^{1}|G(s)|^{2} \mathrm{~d} s \leq \int_{0}^{1}\left|G^{\prime}(s)\right|^{2} \mathrm{~d} s$ and $1=|G(1)|^{2} \leq \int_{0}^{1}\left|G^{\prime}(s)\right|^{2} \mathrm{~d} s$, we obtain:

$$
\delta_{z}^{2} \mu+\left(\frac{\delta_{x}^{2}}{\omega^{2}}-\frac{\epsilon_{i}^{2}+\epsilon_{a}^{2}}{2}\right) v=1
$$

with $0 \leq \mu \leq 1$ and $0 \leq v \leq 1$. 
Imaginary part of the energy equation. Taking the imaginary part of (A.6) and using (A.5)

$$
\mu\left(\epsilon_{a}^{2} \mathfrak{J}\left[\omega^{2}\right]+\delta_{x}^{2} \epsilon_{i}^{2} \mathfrak{J}\left[1 / \omega^{2}\right]\right)+v \delta_{x}^{2} \mathfrak{J}\left[1 / \omega^{2}\right]=0
$$

or, using $\mathfrak{I}\left[1 / \omega^{2}\right]=-\mathfrak{J}\left[\omega^{2}\right] /|\omega|^{4}$,

$$
\mathfrak{J}\left[\omega^{2}\right]\left(\mu\left(\epsilon_{a}^{2}-\epsilon_{i}^{2} \frac{\delta_{x}^{2}}{|\omega|^{4}}\right)-v \frac{\delta_{x}^{2}}{|\omega|^{4}}\right)=0
$$

Let us assume that $\omega$ is neither real or pure imaginary. This implies $\mathfrak{I}\left[\omega^{2}\right] \neq 0$ and (A.7) leads to:

$$
\mu\left(\epsilon_{a}^{2} \frac{|\omega|^{4}}{\delta_{x}^{2}}-\epsilon_{i}^{2}\right)-v=0
$$

We will show below that, in this case, solutions can exist only for non physical values of $\epsilon_{i}, \epsilon_{a}$ satisfying $\max \left(\epsilon_{i}, \epsilon_{a}\right)>\sqrt{2}$.

Real part of the energy equation. Taking the real part of (A.6)

$$
\mu\left(-\delta_{x}^{2}+\delta_{x}^{2} \epsilon_{i}^{2} \mathfrak{R}\left[1 / \omega^{2}\right]-\frac{\left(\epsilon_{i}^{2}+\epsilon_{a}^{2}\right)^{2}}{4}+\epsilon_{a}^{2} \mathfrak{R}\left[\omega^{2}\right]\right)+v\left(\delta_{x}^{2} \mathfrak{R}\left[\frac{1}{\omega^{2}}\right]-\frac{\epsilon_{i}^{2}+\epsilon_{a}^{2}}{2}\right)=1
$$

or, using $\mathfrak{R}\left[1 / \omega^{2}\right]=\mathfrak{R}\left[\omega^{2}\right] /|\omega|^{4}$,

$$
\mu\left(-\delta_{x}^{2}-\frac{\left(\epsilon_{i}^{2}+\epsilon_{a}^{2}\right)^{2}}{4}+\left(\frac{\delta_{x}^{2} \epsilon_{i}^{2}}{|\omega|^{4}}+\epsilon_{a}^{2}\right) \mathfrak{R}\left[\omega^{2}\right]\right)+v\left(\frac{\delta_{x}^{2}}{|\omega|^{4}} \mathfrak{R}\left[\omega^{2}\right]-\frac{\epsilon_{i}^{2}+\epsilon_{a}^{2}}{2}\right)=1 .
$$

(A.8) allows to simplify in:

$$
\mu\left(-\delta_{x}^{2}-\frac{\left(\epsilon_{i}^{2}+\epsilon_{a}^{2}\right)^{2}}{4}+2 \epsilon_{a}^{2} \mathfrak{R}\left[\omega^{2}\right]\right)-v\left(\frac{\epsilon_{i}^{2}+\epsilon_{a}^{2}}{2}\right)=1
$$

Energy-based system of equations. Eqs (A.8) and (A.11) can be summarized in

$$
\begin{aligned}
& \alpha \mu-\beta \nu=1 \\
& \gamma \mu-v=0
\end{aligned}
$$

with

$$
\alpha=-\delta_{x}^{2}-\frac{\left(\epsilon_{i}^{2}+\epsilon_{a}^{2}\right)^{2}}{4}+2 \epsilon_{a}^{2} \mathfrak{R}\left[\omega^{2}\right], \beta=\frac{\epsilon_{i}^{2}+\epsilon_{a}^{2}}{2}, \gamma=\epsilon_{a}^{2} \frac{|\omega|^{4}}{\delta_{x}^{2}}-\epsilon_{i}^{2}
$$

Using $\beta>0$, it is easy to check that (A.12) has solutions $(\mu, v)$ positive and with magnitude less than one only if

$$
\gamma>1 \text { and } \alpha>(1+\beta) \gamma
$$

or

$$
0 \leq \gamma \leq 1 \text { and } \alpha \geq 1+\beta \gamma
$$

The conditions above immediately exclude the cases $\epsilon_{a}=0$ (which leads to $\gamma<0$ ) and $\mathfrak{R}\left[\omega^{2}\right] \leq 0$ (which leads to $\alpha<0$ ). They will not be considered below. 
First case: $0 \leq \gamma \leq 1$. This implies:

$$
|\omega|^{4} \leq \frac{\delta_{x}^{2}}{\epsilon_{a}^{2}}\left(1+\epsilon_{i}^{2}\right)
$$

$\alpha \geq 1+\beta \gamma$ writes:

$$
\mathfrak{R}\left[\omega^{2}\right] \geq \frac{1}{2 \epsilon_{a}^{2}}\left[1+\delta_{x}^{2}+\frac{\left(\epsilon_{a}^{2}+\epsilon_{i}^{2}\right)^{2}}{4}+\frac{\epsilon_{i}^{2}+\epsilon_{a}^{2}}{2}\left(\epsilon_{a}^{2} \frac{|\omega|^{4}}{\delta_{x}^{2}}-\epsilon_{i}^{2}\right)\right]
$$

Now using the inequalities $\left|\mathfrak{R}\left[\omega^{2}\right]\right|^{2} \leq|\omega|^{4}$ and (A.13), we get

$$
\frac{\delta_{x}^{2}}{\epsilon_{a}^{2}}\left(1+\epsilon_{i}^{2}\right) \geq|\omega|^{4} \geq\left(\frac{1}{2 \epsilon_{a}^{2}}\left[1+\delta_{x}^{2}+\frac{\left(\epsilon_{a}^{2}+\epsilon_{i}^{2}\right)^{2}}{4}+\frac{\epsilon_{i}^{2}+\epsilon_{a}^{2}}{2}\left(\epsilon_{a}^{2} \frac{|\omega|^{4}}{\delta_{x}^{2}}-\epsilon_{i}^{2}\right)\right]\right)^{2}
$$

Using a computing algebra software to simplify the technical exercice, we can prove that (A.14) has solutions if and only if

$$
\epsilon_{i}^{2}>\sqrt{4+\epsilon_{a}^{4}}
$$

which requires $\epsilon_{i}>\sqrt{2}$.

Second case: $\gamma>1$. This implies:

$$
|\omega|^{4}>\frac{\delta_{x}^{2}}{\epsilon_{a}^{2}}\left(1+\epsilon_{i}^{2}\right)
$$

$\alpha>(1+\beta) \gamma$ can be written as:

$$
\gamma<\frac{\alpha}{1+\beta}
$$

or

$$
|\omega|^{4}<\frac{\delta_{x}^{2}}{\epsilon_{a}^{2}}\left[\epsilon_{i}^{2}+\frac{1}{1+\frac{\epsilon_{a}^{2}+\epsilon_{i}^{2}}{2}}\left(-\delta_{x}^{2}-\frac{\left(\epsilon_{i}^{2}+\epsilon_{a}^{2}\right)^{2}}{4}+2 \epsilon_{a}^{2} \mathfrak{R}\left[\omega^{2}\right]\right)\right]
$$

Now using $0 \leq \mathfrak{R}\left[\omega^{2}\right] \leq|\omega|^{2}$ and adding (A.15), we get:

$$
\frac{\delta_{x}^{2}}{\epsilon_{a}^{2}}\left(1+\epsilon_{i}^{2}\right)<|\omega|^{4}<\frac{\delta_{x}^{2}}{\epsilon_{a}^{2}}\left[\epsilon_{i}^{2}+\frac{1}{1+\frac{\epsilon_{a}^{2}+\epsilon_{i}^{2}}{2}}\left(-\delta_{x}^{2}-\frac{\left(\epsilon_{i}^{2}+\epsilon_{a}^{2}\right)^{2}}{4}+2 \epsilon_{a}^{2}|\omega|^{2}\right)\right]
$$

which has non trivial solutions if and only if

$$
\epsilon_{a}^{2}>2+\epsilon_{i}^{2}
$$

which requires $\epsilon_{a}>\sqrt{2}$.

Conclusion. This concludes the proof. If $\epsilon_{a}=0$ or $\max \left(\epsilon_{i}, \epsilon_{a}\right) \leq \sqrt{2}$, then $\mathfrak{J}\left[\omega^{2}\right]$ is zero. This also leads to $\mathfrak{J}\left[\delta_{z}^{2}\right]=0$ and we conclude that, under these conditions, $\delta_{z}$ is either real or purely imaginary. Equation (A.10) with $\mu$ and $v$ positive further show that $\left.\operatorname{Re}\left[\omega^{2}\right] \geq 0\right]$ implying that $\omega$ can only be real and the amplitude of the wave solution does not diverge in time. 


\section{A.2 Vanishing ratio $R^{2}\left(\delta_{x}, \delta_{z}\right)$.}

We now show that the two roots $\omega_{ \pm}$are well-separated except when

(i) $\delta_{z}$ is pure imaginary and close to $\delta_{z}=i \delta_{z, i, *}=i\left(\epsilon_{a}^{2}-\epsilon_{i}^{2}\right) / 2$,

(ii) $\delta_{z}$ is real and $\epsilon_{i}=\epsilon_{a} \& \delta_{z}=0$

$\delta_{z}$ real. The ratio of reference functions $R^{2}\left(\delta_{x}, \delta_{z}\right)$, defined by (42), depends indeed on two variables $\left(\delta_{x}, \delta_{z}\right)$ and two parameters $\left(\epsilon_{i}, \epsilon_{a}\right)$. A study of its variations for $\delta_{x} \in \mathbb{R}$ shows that, for non-vanishing $\left(\epsilon_{i}, \epsilon_{a}\right)$, it has an upper bound:

$$
R_{\text {max }}^{2}\left(\delta_{z}\right)=\max _{\delta_{x}} R^{2}\left(\delta_{x}, \delta_{z}\right)=\frac{1}{4} \frac{\epsilon_{a}^{2} \epsilon_{i}^{2}}{\delta_{z}^{2}+\epsilon^{4}}
$$

and this maximum value is attained for

$$
\delta_{x}^{2}=\delta_{z}^{2}+\epsilon^{4}
$$

Since for $\max \left(\epsilon_{i}, \epsilon_{a}\right) \leq \sqrt{2}$ or $\epsilon_{a}=0$ the roots (43) are real (Appendix A.1), $R_{\text {max }}\left(\delta_{z}\right)$ must remain bellow $1 / 4$ but approaches $1 / 4$ for both a near depth-independent vertical profile (i.e. $\delta_{z} \approx 0^{2}$ ) and $\epsilon_{a} \approx \epsilon_{i}$ (or in dimensional form $N=g / c_{s}$ ). This last equality is true when the stratification and the compressibility effects have an identical contribution to the vertical variation of background stratification $\hat{\rho}_{h}(z)$. In all other cases, the vast majority, the two roots are well separated and given by:

$$
\omega_{-}\left(\delta_{x}, \delta_{z}\right) \approx \omega_{i}\left(\delta_{x}, \delta_{z}\right), \quad \omega_{+}\left(\delta_{x}, \delta_{z}\right) \approx \omega_{a}\left(\delta_{x}, \delta_{z}\right)
$$

$\delta_{z}$ pure imaginary. When horizontal and vertical wavenumbers are close together, the left-hand side and right-hand side of (39a) both vanish, i.e., the influence of stratification $\left(\epsilon_{i}^{2} \delta_{x}^{2} / \omega^{2}\right)$ and compressibility $\left(\epsilon_{a}^{2} \omega_{a}^{2}\right)$ cancel out. In other words, differences between horizontal and vertical wavenumbers are indication of the influence of ocean stratification and/or compressibility. In an incompressible, homogeneous (unstratified) ocean, $\epsilon_{i}=\epsilon_{a}=0$ and vertical and horizontal wave-numbers are equal. The developments of $\omega_{-}^{2}, \omega_{+}^{2}$ for small $\epsilon_{i}, \epsilon_{a}$ are identical to those for real vertical wavenumbers (50), (53) just replacing $\delta_{z}^{2}$ by $-\delta_{z, i}^{2}$. The remaining question is that of root separation when $\delta_{z} \in i \mathbb{R}$. Unlike when $\delta_{z} \in \mathbb{R}$ (previous sub-section), the ratio $R^{2}\left(\delta_{x}, i \delta_{z, i}\right)$ can be equal to $1 / 4$ even when $\delta_{z}$ is not small. Relation (39a) imposes $0 \leq \delta_{z, i}^{2} \leq \delta_{x}^{2}+\frac{\left(\epsilon_{a}^{2}+\epsilon_{i}^{2}\right)^{2}}{4}$ and in this range of values, $R^{2}$ is an increasing function of $\delta_{z, i}^{2}$. The value of $R^{2}=1 / 4$ is attained for $\delta_{z, i}^{2}=\delta_{z, i, \star}^{2}$ given by

$$
\delta_{z, i, \star}^{2}=\delta_{x}^{2}+\frac{\left(\epsilon_{a}^{2}+\epsilon_{i}^{2}\right)^{2}}{4}-2 \epsilon_{a} \epsilon_{i} \delta_{x}
$$

for which the inner dispersion relation has a double root

$$
\omega_{+}^{2}=\omega_{-}^{2}=\frac{\epsilon_{i}}{\epsilon_{a}} \delta_{x}
$$

When $\delta_{z, i}$ is less than $\delta_{z, i, \star}$, the two roots become separated. Again, since we have proved in appendix that only solutions with real angular frequency can exist (when the domain is bounded), the case $\delta_{z, i}^{2}>\delta_{z, i, \star}^{2}$, which would lead to $R^{2}>1 / 4$ can be excluded. Note that $\omega_{-}, \omega_{+}$as functions of $\delta_{z, i}$ are not differentiable at the neutral point $\delta_{z, i}=\delta_{z, i, \star}$.

\footnotetext{
${ }^{2}$ We use the term depth-independent even if $F(z)$ is a linear function of $z$ when $\delta_{z}$ is close to 0 (see (28)), so that the vertical profile of $W(z)$ is approximately linear while the vertical profile of $U(z)$ is approximately constant.
} 


\section{A.3 Internal and acoustic modes (MIM \& MAM).}

MIM solutions: we have shown in $\S(3.4)$ that the root of the inner dispersion relation corresponding to internal gravity waves is well-approximated by $\omega_{i}^{2}$. Making $\omega^{2}\left(\delta_{x}\right) \approx \omega_{i}^{2}$ in the boundary dispersion relation $(34 \mathrm{~b})$ leads to

$$
\frac{1}{\delta_{z} / \tan \left(\delta_{z}\right)+\frac{\epsilon_{i}^{2}+\epsilon_{a}^{2}}{2}} \approx \frac{\epsilon_{i}^{2}}{\delta_{x}^{2}+\delta_{z}^{2}+\frac{1}{4}\left(\epsilon_{i}^{2}+\epsilon_{a}^{2}\right)^{2}}
$$

If we assume here that $\delta_{z}$ is not close to 0 , the right-hand side of (A.21) is always small and thus $\delta_{z} / \tan \left(\delta_{z}\right)$ has to be large, i.e., the vertical wavenumber $\delta_{z}$ is close to $\delta_{z, n} \approx n \pi$ with $n$ a non-zero integer. This agrees with the internal gravity wave solution found graphically in subsection (4.2).

MAM solutions: we can now use the fact that the root of the inner dispersion relation corresponding to acoustic waves is well-approximated by $\omega_{a}^{2}$. Making $\omega^{2}\left(\delta_{x}\right) \approx \omega_{a}^{2}$ in the boundary dispersion relation (34b) leads to:

$$
\delta_{z} / \tan \left(\delta_{z}\right)+\frac{\epsilon_{i}^{2}+\epsilon_{a}^{2}}{2} \approx \frac{\epsilon_{a}^{2}}{1+\left(\delta_{z}^{2}+\frac{1}{4}\left(\epsilon_{i}^{2}+\epsilon_{a}^{2}\right)^{2}\right) / \delta_{x}^{2}}
$$

Since the right-hand side of (A.22) is small (bounded by $\epsilon_{a}^{2}$ ), $\delta_{z} / \tan \left(\delta_{z}\right)$ has to be small and thus the vertical wavenumber $\delta_{z}$ is close to $\delta_{z, m} \approx \pi / 2+m \pi$, with $m \in \mathbb{N}$.

\section{A.4 Surface waves existence.}

In the more general case of a stratified and compressible ocean, we now show the existence of solutions to (39a) and (39b) the dispersion relations for $\delta_{z} \in i \mathbb{R}$.

Let us define $f\left(\delta_{x}, \delta_{z, i}\right)$ by:

$$
f\left(\delta_{x}, \delta_{z, i}\right)=\delta_{x}^{2}-\delta_{z, i}^{2}-\left(\epsilon_{i}^{2} \frac{\delta_{x}^{2}}{\omega^{2}}+\epsilon_{a}^{2} \omega^{2}-\frac{\left(\epsilon_{a}^{2}+\epsilon_{i}^{2}\right)^{2}}{4}\right), \quad \text { with } \omega^{2}=\frac{\delta_{x}^{2}}{\frac{\delta_{z, i}}{\tanh \left(\delta_{z, i}\right)}+\frac{\epsilon_{a}^{2}+\epsilon_{i}^{2}}{2}}
$$

The inner boundary relation translates into $f\left(\delta_{x}, \delta_{z, i}\right)=0$. We can check that, for a given $\delta_{z, i}, f\left(\delta_{x}, \delta_{z, i}\right)$ is an increasing function of $\delta_{x}{ }^{3}$. Let $\delta_{x, 0}$ be the value of $\delta_{x}$ defined by $f\left(\delta_{x, 0}, \delta_{z}=0\right)=0\left(\delta_{x, 0}\right.$ will be given in (64), and is close to $\left.\epsilon_{i}\right)$. In particular, we have $f\left(\delta_{x}, 0\right) \leq f\left(\delta_{x, 0}, 0\right)=0$ for $\delta_{x} \leq \delta_{x, 0}$. We can also verify that for a given $\delta_{x}$ such that $\delta_{x} \leq \delta_{x, 0}$, the maximum of $f\left(\delta_{x}, \delta_{z}\right)$ is attained in $\delta_{z, i}=0$. This proves that if $\delta_{x}<\delta_{x, 0}, f\left(\delta_{x}, \delta_{z, i}\right)<0 \forall \delta_{z, i}$. Therefore, modified surface waves can only exist under the condition $\delta_{x} \geq \delta_{x, 0}$.

\section{A.5 Surface waves: $\delta_{z, i} \approx \delta_{x}$.}

For small parameters $\epsilon_{a}$ and $\epsilon_{i}$, we show in the present section that $\delta_{z, i}^{2}$ is close to $\delta_{x}^{2}$ for any MSW solution.

We can show that $1 \leq \frac{\delta_{z, i}}{\tanh \left(\delta_{z, i}\right)} \leq \delta_{z, i}^{2}+1, \quad \forall \delta_{z, i} \in \mathbb{R}$. Injecting these two inequalities in the surface

${ }^{3}$ This simple demonstration requires the assumption $\epsilon_{a}^{2} \leq 2+\epsilon_{i}^{2}$, i.e., the same used in appendix to prove that the imaginary part of $\delta_{z}^{2}$ is zero. 
dispersion relation leads to:

$$
\frac{\delta_{x}^{2}}{\omega^{2}} \leq \delta_{z, i}^{2}+1+\frac{\epsilon_{a}^{2}+\epsilon_{i}^{2}}{2}, \quad \text { and } \quad \omega^{2} \leq \delta_{x}^{2}
$$

Since the inner dispersion relation implies $-\frac{\left(\epsilon_{a}^{2}+\epsilon_{i}^{2}\right)^{2}}{4} \leq \delta_{x}^{2}-\delta_{z, i}^{2} \leq \epsilon_{i}^{2} \frac{\delta_{x}^{2}}{\omega^{2}}+\epsilon_{a}^{2} \omega^{2}$, we finally get:

$$
-\frac{\left(\epsilon_{a}^{2}+\epsilon_{i}^{2}\right)^{2}}{4} \leq \delta_{x}^{2}-\delta_{z, i}^{2} \leq \epsilon_{i}^{2} \delta_{z, i}^{2}+\epsilon_{a}^{2} \delta_{x}^{2}+\epsilon_{i}^{2}\left(1+\frac{\epsilon_{a}^{2}+\epsilon_{i}^{2}}{2}\right)
$$

Using the smallness of the parameters $\epsilon_{a}$ and $\epsilon_{i}$, we can then conclude that $\delta_{z, i}^{2}$ is indeed close to $\delta_{x}^{2}$.

\section{A.6 MIM0 long-wave sub-branch}

In the present section of the appendix, we show that the horizontal wave-number of the MIM0 solution $\left(\delta_{x, \operatorname{mim} 0}\left(\delta_{z}\right), \omega_{\text {mim } 0}\left(\delta_{z}\right)\right)$ is a decreasing function of $\delta_{z}$. It satisfies $\delta_{x, \operatorname{mim} 0}(0)=\delta_{x, 0} \geq 0$ and $\delta_{x, \operatorname{mim} 0}\left(\delta_{z, 0}\right)=0$ for $\delta_{z, 0} \in\left[0, \delta_{z, m=0} \approx \pi / 2[\right.$.

Wave solutions of MIM0 type must satisfy both the inner and boundary dispersion relation (34a and 34b) with $\delta_{z} \in \mathbb{R}$. We have also shown that MIM0 is part of the internal branch and as a consequence approximately satisfies (41b). Substituting then (34b) into (41b) leads to a relation between the horizontal and vertical wave-numbers:

$$
\delta_{x}^{2}=\delta_{x, \operatorname{mim} 0}^{2}\left(\delta_{z}\right)=-\delta_{z}^{2}-\epsilon^{4}+\epsilon_{i}^{2}\left(\epsilon^{2}+\delta_{z} \operatorname{cotan}\left(\delta_{z}\right)\right)
$$

with $\delta_{x, \text { mim } 0}^{2}(0)=\delta_{x, 0}^{2}=-\epsilon^{4}+\epsilon_{i}^{2}\left(\epsilon^{2}+1\right)$. When $\delta_{x, 0}^{2}<0$, this depth-independent solution does not propagate horizontally and we will assume in the following that $\delta_{x, 0}^{2}=-\epsilon^{4}+\epsilon_{i}^{2}\left(\epsilon^{2}+1\right) \geq 0$. In $\S(4.2)$, $\delta_{z, m=0}$ has been defined as a root of $\epsilon^{2}+\delta_{z} \operatorname{cotan}\left(\delta_{z}\right)$ and when $\delta_{z} \rightarrow \delta_{z, m=0}^{(-)}, \delta_{x}^{2}\left(\delta_{z}\right) \rightarrow-\infty$. This means that there must exist $\delta_{z, 0} \in\left[0, \delta_{z, m=0}\left[\right.\right.$ such that $\delta_{x, \operatorname{mim} 0}\left(\delta_{z, 0}\right)=0$ and $\delta_{z, 0}$ satisfies:

$$
-\delta_{z}^{2}-\epsilon^{4}+\epsilon_{i}^{2}\left(\epsilon^{2}+\delta_{z} \operatorname{cotan}\left(\delta_{z}\right)\right)=0
$$

We can additionally show that $\delta_{x, \operatorname{mim} 0}\left(\delta_{z}\right)$ is monotonic (decreasing) for $\delta_{z} \in\left[0, \delta_{z, 0}\right]$. Indeed, its derivative

$$
\frac{d \delta_{x, \operatorname{mim} 0}}{d \delta_{z}}\left(\delta_{z}\right)=2 \delta_{z}^{2}+\epsilon_{i}^{2}\left(\delta_{z}-\operatorname{cotan}\left(\delta_{z}\right)\right)
$$

is positive for $\delta_{z} \in\left[0, \delta_{z, 0}\right]$ since it satisfies $\frac{d \delta_{x, m i m 0}}{d \delta_{z}}(0)=0$ and is an increasing function of $\delta_{z}$ over this interval. The monotonicity of the derivative can be proven by computing the second order derivative

$$
\frac{d^{2} \delta_{x, \operatorname{mim} 0}}{d \delta_{z}^{2}}\left(\delta_{z}\right)=2+2 \epsilon_{i}^{2} \frac{1-\delta_{z} \operatorname{cotan}\left(\delta_{z}\right)}{\sin ^{2}\left(\delta_{z}\right)}
$$

and using the Taylor series of $\operatorname{cotan}(z)$, we can further show that $\left(1-\delta_{z} \operatorname{cotan}\left(\delta_{z}\right)\right) \geq 0$ and thus $\frac{d^{2} \delta_{x, m i m 0}}{d \delta_{z}^{2}}\left(\delta_{z}\right) \geq 0$. 


\section{A.7 Monotonicity of the branches of wave solutions ?}

In the present section of the appendix, we show that the branches of acoustic wave solutions are monotonic and decreasing if $\epsilon_{a}$ and $\epsilon_{i}$ remain small.

To do so, the derivatives of $\delta_{x}^{2}$ and $\omega^{2}$ with respect to $\delta_{z}$ given respectively by (55a) and (55b) are computed:

$$
\begin{aligned}
\left(\epsilon^{2}-J\left(\delta_{z}\right)\right)^{2} \frac{d \delta_{x}^{2}}{d \delta_{z}}\left(\delta_{z}\right)= & -2 \delta_{z} J^{2}\left(\delta_{z}\right) \\
& +2 \epsilon^{2} \delta_{z}^{2} J^{\prime}\left(\delta_{z}\right)+\epsilon_{i}^{2} J^{2}\left(\delta_{z}\right) J^{\prime}\left(\delta_{z}\right) \\
& +2 \epsilon^{4} \delta_{z}-\left(\epsilon_{i}^{4}+\epsilon_{i}^{2} \epsilon_{a}^{2}\right) J\left(\delta_{z}\right) J^{\prime}\left(\delta_{z}\right) \\
& +\frac{1}{4}\left(\epsilon_{a}^{6}-2 \epsilon_{i}^{6}-3 \epsilon_{i}^{4} \epsilon_{a}^{2}\right) J^{\prime}\left(\delta_{z}\right) \\
\left(\epsilon^{2}-J\left(\delta_{z}\right)\right)^{2} \frac{d \omega^{2}}{d \delta_{z}}\left(\delta_{z}\right)= & \delta_{z}^{2} J^{\prime}\left(\delta_{z}\right)-2 \delta_{z} J\left(\delta_{z}\right) \\
& +\left(\epsilon_{a}^{2}-\epsilon_{i}^{2}\right) \delta_{z}^{2} \\
& +\frac{1}{4}\left(\epsilon_{a}^{4}-3 \epsilon_{i}^{4}-2 \epsilon_{a}^{2} \epsilon_{i}^{2}\right) J^{\prime}\left(\delta_{z}\right)
\end{aligned}
$$

where

$$
\begin{aligned}
& J\left(\delta_{z}\right)=\delta_{z} \operatorname{cotan}\left(\delta_{z}\right) \\
& J^{\prime}\left(\delta_{z}\right)=\frac{\sin \left(2 \delta_{z}\right)-2 \delta_{z}}{2 \sin ^{2}\left(\delta_{z}\right)} \leq 0
\end{aligned}
$$

Note that the relations above are not Taylor expansions of the derivatives with respect to $\epsilon_{a}$ and $\epsilon_{i}$, they are exact relations. The derivative of $\delta_{x}^{2}$ given by (A.27a) is negative at third order in $\left(\epsilon_{a}, \epsilon_{i}\right)$ whereas the derivative of $\omega^{2}$ (A.27b) is negative only at first order in $\left(\epsilon_{a}, \epsilon_{i}\right)$. At higher order, (A.27a) and (A.27b) additionally show the values of $\delta_{z}$ for which $\delta_{x}^{2}$ and $\omega^{2}$ are monotonic depending of the values of this two parameters.

$\delta_{x}^{2}\left(\delta_{z}\right)$ and $\omega^{2}\left(\delta_{z}\right)$ are thus monotonously decreasing functions of $\delta_{z}$ if $\epsilon_{a}$ and $\epsilon_{i}$ remains small.

\section{B Incompressible hydrostatic model \& non-hydrostatic models}

Hydrostatic \& incompressible model. Under the hydrostatic assumption, the propagation relation (16) in a uniformly stratified, incompressible ocean turns to:

$$
\tilde{W}^{\prime \prime}(z)+\frac{g}{D_{0} \omega} \tilde{W}(z)=0
$$

associated to the surface and bottom boundary conditions:

$$
\tilde{W}^{\prime}(0)-\frac{g k_{x}^{2}}{\omega^{2}} \tilde{W}(0)=0, \quad \tilde{W}(-H)=0
$$

The inner and boundary dispersion relations are then:

$$
\begin{aligned}
& \delta_{z}^{2}-\frac{\epsilon_{i}^{2} \delta_{x}^{2}}{\omega^{2}}=0 \\
& \omega^{2}=\frac{\delta_{x}^{2} \tan \left(\delta_{z}\right)}{\delta_{z}}
\end{aligned}
$$

In the long wave-approximation $\left(\delta_{z} \rightarrow 0\right)$, (B.3a) and (B.3b) are approximately given by $\left(\delta_{z}=\epsilon_{i}, \omega=\delta_{x}\right)$ and tends to the shallow-water solution $\left(\delta_{z}=0, \omega=\delta_{x}\right)$ (SHW, table 1) for a homogeneous ocean $\left(\epsilon_{i} \rightarrow 0\right)$. 
Non-hydrostatic \& incompressible model. In an incompressible (non-hydrostatic) ocean, the propagation relation (16) in a uniformly stratified, incompressible ocean turns to:

$$
\tilde{W}^{\prime \prime}(z)+k x^{2}\left(\frac{g}{D_{0} \omega}-1\right) \tilde{W}(z)=0
$$

associated to the surface and bottom boundary conditions:

$$
\tilde{W}^{\prime}(0)-\frac{g k_{x}^{2}}{\omega^{2}} \tilde{W}(0)=0, \quad \tilde{W}(-H)=0
$$

The inner and boundary dispersion relations are then:

$$
\begin{aligned}
& \delta_{x}^{2}+\delta_{z}^{2}-\frac{\epsilon_{i}^{2} \delta_{x}^{2}}{\omega^{2}}=0 \\
& \omega^{2}=\frac{\delta_{x}^{2} \tan \left(\delta_{z}\right)}{\delta_{z}}
\end{aligned}
$$

In the long wave-approximation $\left(\delta_{z} \rightarrow 0\right)$, (B.6a) and (B.6b) are approximately given by $\left(\delta_{z}=\delta_{x}+\right.$ $\left.\epsilon_{i}^{2}, \omega=\delta_{x}\right)$ and tends to the long-swell solution $\left(\delta_{z}=\delta_{x}, \omega=\delta_{x}\right)$ (LSW, table 1) in a homogeneous ocean $\left(\epsilon_{i} \rightarrow 0\right)$.

Dispersion surfaces. Figure (B.1) shows the inner and boundary dispersion surfaces for an incompressible ocean under the assumptions of hydrotaticity (a) and non-hydrostaticity (b).

- Under the hydrostatic assumption, all wave solution have real, positive vertical number $\delta_{z}$. Figure (B.1.a) shows two types of solutions, MIM for $\delta_{z} \approx \pi$ and barotropic-like mode for small $\delta_{z}$ which converge to barotropic mode when $\epsilon_{i} \rightarrow 0$.

- Under the non-hydrostatic assumption, Figure (B.1.b) shows also two types of solutions, MIM for $\delta_{z} \approx \pi$ and MSW for $\delta_{z} \in i \mathbb{R}$.

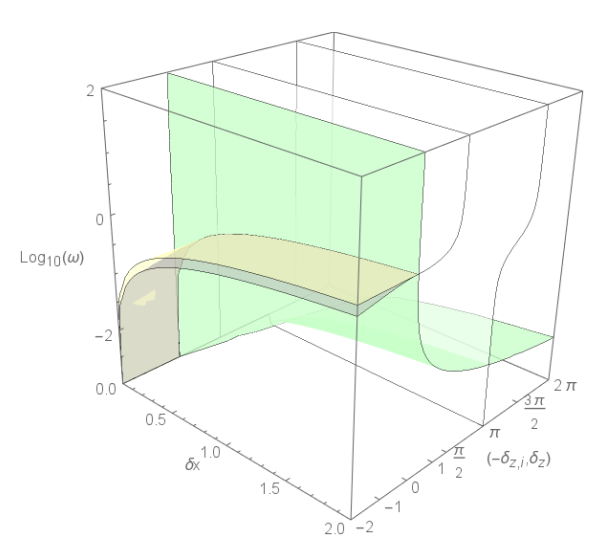

(a)

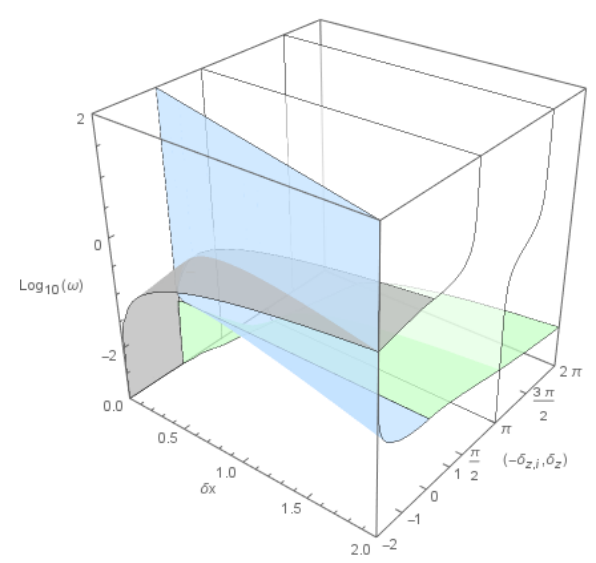

(b)

Figure B.1: inner and boundary dispersion surfaces in $\left(\delta_{x}, \delta_{z}, \omega\right)$ space and wave solutions for an incompressible ocean:

(a) hydrostatic assumption,

(b) non-hydrostatic assumption.

Colors: (light red) $\delta_{z}^{2} \geq 0 \mathcal{E} \omega \geq 1$, (light green) $\delta_{z}^{2} \geq 0 \mathcal{E} \omega<1$, (light blue) $\delta_{z}^{2}<0$, (white) boundary dispersion surface for $\delta_{z} \in \mathbb{R}$, (light gray) boundary dispersion surface for $\delta_{z} \in i \mathbb{R}$, (light yellow) $\omega^{2}=\delta_{x}^{2}$ barotropic wave. 


\section{Analytical solutions and vertical profiles of vertical velocity under constant Brunt- Väisälä angular frequency}

Analytical solutions. We define in $\S(2)$ the vertical velocity profile $\widetilde{W}(z)$ for a constant and uniform Brunt-Väsälä angular frequency by

$$
\widetilde{W}(z)=e^{-z /\left(2 D_{0}\right)} F(z)=e^{-z / 2 D_{0}} \frac{\sin \left(k_{z}(H+z)\right)}{\sin \left(k_{z} H\right)}
$$

and a vertical pressure profile $\widetilde{p}(z)$

$$
\widetilde{p}(z)=\frac{\Omega^{2}}{g^{2} k_{x}^{2}\left(1-\left(\Omega /\left(c_{s} k_{x}\right)\right)^{2}\right)}\left(N_{0}^{2} \widetilde{W}(z)+g \widetilde{W}^{\prime}(z)\right) .
$$

with $\frac{1}{D_{0}}=\frac{N_{0}^{2}}{g}+\frac{g}{c_{s}^{2}}$, and where, for a given horizontal wave-number $k_{x}, k_{z}$ and $\Omega$ are solutions of the inner and boundary dispersion relations. Highly accurate approximations of them can be found in table (5).

Note that by construction (and using the boundary dispersion relation), we have $\widetilde{W}(0)=\widetilde{p}(0)=1$.

The real analytical solutions of Eqs. (13a, 13b, 13c, 13d) which satisfy the bottom and surface conditions (14a, 14b) are given by:

$$
\begin{aligned}
\eta(x, t) & =\eta_{0} \cos \left(k_{x} x\right) \cos (\Omega t) \\
u(x, z, t) & =\eta_{0} \frac{g k_{x}}{\Omega} \sin \left(k_{x} x\right) \sin (\Omega t) \frac{\hat{\rho}_{h}(0)}{\hat{\rho}_{h}(z)} \widetilde{p}(z) \\
w(x, z, t) & =-\eta_{0} \Omega \cos \left(k_{x} x\right) \sin (\Omega t) \frac{\hat{\rho}_{h}(0)}{\hat{\rho}_{h}(z)} \widetilde{W}(z) \\
p(x, z, t) & =\eta_{0} g \hat{\rho}_{h}(0) \cos \left(k_{x} x\right) \cos (\Omega t) \widetilde{p}(z) \\
\rho(x, z, t) & =\eta_{0} \hat{\rho}_{h}(0) \cos \left(k_{x} x\right) \cos (\Omega t)\left(\frac{N_{0}^{2}}{g} \widetilde{W}(z)+\frac{g}{c_{s}^{2}} \widetilde{p}(z)\right)
\end{aligned}
$$

with $\hat{\rho}_{h}(z)=\hat{\rho}_{h}(0) e^{-z / D_{0}}$ and $\eta_{0}$ a constant.

For an homogeneous $N_{0}^{2} \rightarrow 0$ and incompressible $\left(c_{s} \rightarrow \infty\right.$, and thus $\left.D_{0} \rightarrow \infty\right)$ non hydrostatic ocean, the Airy's solutions are recovered (see e.g. (Gill 1982, section 5.3)). Indeed $k_{z}=i k_{x}, \Omega^{2}=g k_{x} \tanh \left(H k_{x}\right)$ and

$$
\widetilde{W}_{\text {Airy }}(z)=F_{\text {Airy }}(z)=\frac{\sinh \left(k_{x}(H+z)\right)}{\sinh \left(k_{x} H\right)}, \quad \widetilde{p}_{\text {Airy }}(z)=\frac{\Omega^{2}}{g k_{x}^{2}} \widetilde{W}_{\text {Airy }}^{\prime}(z)=\frac{\Omega^{2}}{g k_{x}} \frac{\cosh \left(k_{x}(H+z)\right)}{\sinh \left(k_{x} H\right)},
$$

leading to

$$
\begin{aligned}
\eta_{\text {Airy }}(x, t) & =\eta_{0} \cos \left(k_{x} x\right) \cos (\Omega t) \\
u_{\text {Airy }}(x, z, t) & =\eta_{0} \Omega \sin \left(k_{x} x\right) \sin (\Omega t) \frac{\cosh \left(k_{x}(H+z)\right)}{\sinh \left(k_{x} H\right)} \\
w_{\text {Airy }}(x, z, t) & =-\eta_{0} \Omega \cos \left(k_{x} x\right) \sin (\Omega t) \frac{\sinh \left(k_{x}(H+z)\right)}{\sinh \left(k_{x} H\right)} \\
p_{\text {Airy }}(x, z, t) & =\hat{\rho}_{h}(0) g \eta_{0} \cos \left(k_{x} x\right) \cos (\Omega t) \frac{\cosh \left(k_{x}(H+z)\right)}{\cosh \left(k_{x} H\right)} \\
\rho_{\text {Airy }}(x, z, t) & =0
\end{aligned}
$$

Vertical profiles. Figure (C.1) shows the several vertical profiles of the vertical velocity for several propagating MSW, MIM0 and MIM1 solutions and vanishing MIM0 solutions $\left(\omega^{2}<0\right.$ and $\left.\delta_{x}^{2}<0\right)$. For short-length MSW $\left(\delta_{x}>\delta_{x, 0}\right)$, the vertical profile of the vertical (blue curves) is concave, it is linear for $\delta_{x}=\delta_{x, 0}$ and convex for long-waves $\left(\delta_{x}<\delta_{x, 0}\right)$. the vertical profiles of MIM1 and MAM1 (MIM2 and MAM2) show one (respectively two) extrema in the water column. 


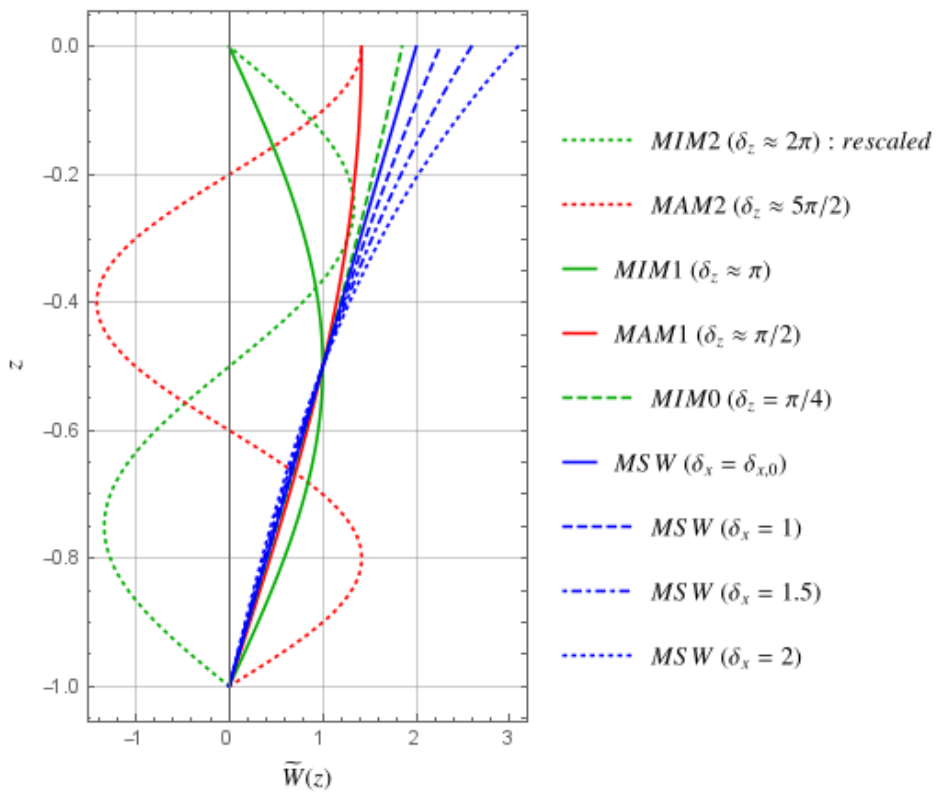

Figure C.1: vertical profiles of vertical velocity $\widetilde{W}(z)$. Green curves: MIM solutions. Red curves: MAM solutions. Blue curves: MSW solutions. 